\title{
Two-dimensional $\operatorname{ReS}_{2}$ : Solution to the unresolved queries on its structure and inter-layer coupling leading to potential optical applications
}

\author{
Janardhan Rao Gadde, ${ }^{1, *}$ Anasuya Karmakar, ${ }^{2, *}$ Tuhin Kumar Maji, ${ }^{3,{ }^{*}}$ Subhrajit Mukherjee, ${ }^{4}$ Rajath Alexander, ${ }^{5}$ \\ Anjanashree M. R. Sharma, ${ }^{6}$ Sarthak Das, ${ }^{7}$ Anirban Mandal, ${ }^{8}$ Kinshuk Dasgupta $\odot,{ }^{5}$ Akshay Naik, ${ }^{6}$ Kausik Majumdar, ${ }^{7}$ \\ Ranjit Hawaldar, ${ }^{1}$ K. V. Adarsh, ${ }^{8}$ Samit Kumar Ray, ${ }^{4,9}$ and Debjani Karmakar $\oplus^{10, \dagger}$ \\ ${ }^{1}$ Centre for Materials for Electronic Technology, Pune 411008, India \\ ${ }^{2}$ Indian Institute of Science Education and Research, Pune 411008, India \\ ${ }^{3}$ Department of Chemical Biological and Macromolecular Sciences, S. N. Bose National Centre for Basic Sciences, \\ Salt Lake, Kolkata 700106, India \\ ${ }^{4}$ Department of Physics, Indian Institute of Technology Kharagpur, Kharagpur 721302, India \\ ${ }^{5}$ Advanced Carbon Materials Section, Materials Science Group, Bhabha Atomic Research Centre, Trombay, Mumbai 400085, India \\ ${ }^{6}$ Centre for Nano Science and Engineering, Indian Institute of Science, Bangalore 560012, India \\ ${ }^{7}$ Department of Electrical Communication Engineering, Indian Institute of Science, Bangalore 560012, India \\ ${ }^{8}$ Department of Physics, Indian Institute of Science Education and Research, Bhopal 462066, India \\ ${ }^{9}$ Department of Condensed Matter Physics and Material Science, S. N. Bose National Centre for Basic Sciences, \\ Salt Lake, Kolkata 700106, India \\ ${ }^{10}$ Technical Physics Division, Bhabha Atomic Research Centre, Trombay, Mumbai 400085, India
}

(Received 4 February 2021; revised 9 April 2021; accepted 27 April 2021; published 17 May 2021)

\begin{abstract}
Over the last few years, $\operatorname{ReS}_{2}$ has generated a myriad of unattended queries regarding its structure, the concomitant thickness-dependent electronic properties, and its apparently contrasting experimental optical response. In this paper, with elaborate first-principles investigations, using density functional theory (DFT) and time-dependent DFT, we identify the structure of $\mathrm{ReS}_{2}$, which is capable of reproducing and analyzing the layer-dependent optical response. The theoretical results are further validated by an in-depth structural, chemical, optical, and optoelectronic analysis of the large-area $\operatorname{ReS}_{2}$ thin films, grown by the chemical vapor deposition (CVD) process. Micro-Raman, x-ray photoelectron spectroscopy, cross-sectional transmission electron microscopy, and energy-dispersive $\mathrm{x}$-ray analysis have enabled the optimization of the uniform growth of the CVD films. The correlation between the optical and electronic properties was established by static photoluminescence and excited state transient absorption measurements. Sulfur vacancy-induced localized mid-gap states render a significantly long lifetime of the excitons in these films. The ionic gel top-gated photodetectors, fabricated from the as-prepared CVD films, exhibit a large photo-response of $\sim 5 \mathrm{~A} / \mathrm{W}$ and a remarkable detectivity of $\sim 10^{11}$ Jones. The outcome of this paper will be useful in promoting the application of vertically grown large-area films in the field of optics and opto-electronics.
\end{abstract}

DOI: 10.1103/PhysRevMaterials.5.054006

\section{INTRODUCTION}

The transition metal dichalcogenide (TMDC) $\mathrm{ReS}_{2}$ had initially instigated a lot of expectations in the fields of optics and opto-electronics because of the prior prediction of its layer-independent electronic properties and thereby inferred direct band gap from bulk to the monolayer [1]. Few-layered $\mathrm{ReS}_{2}$ had shown a reasonable photo-response, stimulating a panoply of applications in photodetectors [2-4], phototransistors [5], logic circuits [6-8], photocatalysis [9,10], and so on. $\mathrm{ReS}_{2}$ differs from the common TMDC systems by virtue of its strongly anisotropic polarization-dependent optical [11-16],

\footnotetext{
*These authors contributed equally to this work.

Corresponding author: debjan@barc.gov.in
}

excitonic [17-20], and thermal responses [2,21-23], originating from its lower intra-plane symmetries in the distorted $1 \mathrm{~T}^{\prime}$ structural format.

The system has continued to remain a source of a multitude of controversies over the last few years since its structure, as predicted by Murray et al. [24] and Wildervanck and Jellinek [25], does not corroborate the electronic properties derived from a series of optical experiments, performed mostly on two-dimensional (2D) exfoliated single crystals and thin films with varying layer thicknesses. Although the x-ray diffraction measurements of the halide-assisted high-temperature-grown bulk single crystals by Ho et al. [26] had indicated the structure defined by Wildervanck and Jellinek [25], the structural confirmation of 2D low-temperature growth of $\mathrm{ReS}_{2}$ is still under cogitation and altercation. The initial studies on $\mathrm{ReS}_{2}$ predict the additional electron in $\mathrm{Re}$ to be responsible for the strong intra-plane Re-Re metallic bonds. 
Such metallic bonding leads to the formation of Peierls distorted Re4 parallelogram array along an in-plane axis. As a result, there is a consequential and significant increase of the intra-plane interactions, rendering the inter-plane couplings negligible [1]. These studies predict $\mathrm{ReS}_{2}$ to be a direct band gap semiconductor irrespective of its layer thicknesses [1]. The subsequent experimental studies, however, strongly oppose these predictions about the structural and electronic properties, revealing strong layer-dependent optical [11,27] and vibrational [28] properties of $\operatorname{ReS}_{2}$. An additional indication of lesser quantum yields of photoluminescence (PL) with lowering thicknesses [29] also contradicts the direct band gap nature of monolayer and few-layered $\mathrm{ReS}_{2}$. The optical response divulges that, starting from the monolayer up to seven layers, $\operatorname{ReS}_{2}$ remains an indirect band gap semiconductor [28]. A couple of recent angle-resolved photoemission studies on bulk and few-layered $\mathrm{ReS}_{2}$ have unfolded a significant delocalization of valence electrons over the inter-layer van der Waals gap, nullifying the prediction of negligible inter-layer interactions [30-32]. Starting from the structure predicted by Murray et al. [24], it has also been demonstrated that, except bilayer $\mathrm{ReS}_{2}$, all other layered $\mathrm{ReS}_{2}$ systems possess an indirect band gap [30]. Moreover, few-layered $\mathrm{ReS}_{2}$ exhibits a series of inter-layer shear and breathing modes at frequencies $<50 \mathrm{~cm}^{-1}$ [33-35], strengthening the probability of a strong inter-layer coupling in $\mathrm{ReS}_{2}$. Therefore, the understanding of the correlation between the structural, optical, and electronic properties of $\mathrm{ReS}_{2}$ does not have the requisite transparency, despite the existence of numerous studies in prior literature.

An exhaustive survey of the prior results has indicated the existence of two possible structures of $\mathrm{ReS}_{2}$ in the literature. The cell parameters, unit cell orientations, and polyhedral Re-S coordination of these two structures are widely different. The structure defined by Murray et al. [24] possesses a distorted octahedral (OCT) coordination and ignores one center of reflection. Later on, Lamfers et al. [36] have suggested the correct structure of $\mathrm{ReS}_{2}$, with two types or polyhedral coordination, modified cell parameters, and unit-cell orientation, where they claimed to have rectified the errors in the structure of Murray et al. [24]. Interestingly, all prior theoretical studies have concentrated only on the structure by Murray et al. [24], and the structural rectification suggested by Lamfers et al. [36] remained apparently unattended.

In this paper, we aim at filling up the existing voids in the understanding of the electronic properties of $\mathrm{ReS}_{2}$ with the help of theoretical analysis and subsequent experimental validations. The organization of this paper is as follows. In the next section, we have furnished a comparison between two different structural configurations of $\mathrm{ReS}_{2}$ available in the literature and, with a thorough first-principles investigation, have identified the correct one capable of reproducing the experimental results. Subsequently, synthesis of large-area thin film was carried out, and a stoichiometric optimization of the CVD growth process was obtained with the help of spectroscopic characterizations, as described in the next section. As a next step, well-characterized as-grown large-area systems are utilized for demonstrating optical and opto-electronic applications. The last section provides a summary and conclusion of the obtained results.

\section{THEORETICAL ANALYSIS: CORRELATION BETWEEN STRUCTURE AND PROPERTIES}

$\mathrm{ReS}_{2}$ belongs to the triclinic symmetry group $P^{1}$, resembling distorted $\mathrm{CdCl}_{2}$ structure. Intricate scrutiny of prior literature regarding its structure leads to two different results. The structure defined by Murray et al. [24], having four formula units per unit cell, is rectified by Lamfers et al. [36], with a modified orientation of the unit cell, numerical values of the cell parameters, and the underlying inversion symmetry. Throughout this paper, we will define the structures described by Lamfers et al. [36] and Murray et al. [24] as S-1 and S-2, respectively. The $\mathrm{S}-1$, possessing eight formula units per unit cell, has four almost coplanar independent crystallographic sites for Re due to the pseudo-inversion center at $\left(\frac{1}{4}, 0,0\right)$, the repetition of which along the $c$ axis is related by a weak reflection symmetry. This center of reflection was ignored at $\mathrm{S}-2$, leading to a halving of its $c$ axis. In addition, for the orientation of the unit cell in $\mathrm{S}-2$, the $\mathrm{ReS}_{2}$ planes are stacked along the $a$ axis, in contrast to the $c$-axis stacking derived for S-1. A pictorial comparison of these two structures and their corresponding unit cell orientations is presented in Fig. 1. As per our knowledge, maximal prior studies refer to the structure as in S-2, and the structure defined by S-1 remains disregarded to date. In this paper, we have compared the energetics and the electronic band properties corresponding to these two structures.

The main characteristic, which differentiates these two structures, is their respective polyhedral coordination. For S1 , there are two types of polyhedra. On the $a-b$ plane, the face-shared distorted $\mathrm{ReS}_{6}$ OCT chains along the $b$ axis are bridged by the two face-shared couplets of $\mathrm{ReS}_{5}$ distorted square pyramids (SQP) along the $a$ axis. Figures 1(a) and 1(c) present a comparison of the polyhedral coordination and the corresponding unit cell orientations of S-1 and S-2, respectively. There is only one type of polyhedral coordination in $\mathrm{S}-2$, where the face-shared OCT pervades the $b-c$ plane. The Re4 parallelogram chains, as shown within the highlighted ellipse, are aligned along the $a$ and $b$ axes for $\mathrm{S}-1$ and S-2, respectively [Figs. 1(b) and 1(d)].

Comparative analysis of the electronic structures of both S-1 and S-2 was carried out by first-principles calculations using the generalized gradient approximation with a hybrid BLYP exchange correlation functional, after incorporating the spin-orbit coupling. The details of the parameters of the theoretical calculations are presented in the Supplemental Material [37]. To compare the formation energies of S-1 and $\mathrm{S}-2$, we have taken a $1 \times 1 \times 2$ supercell of S-1 and a similar supercell of S-2, constructed from its $a-c$ flipped unit cell. The formation energies of both structures, calculated by using the formula $E_{\text {form }}=E\left(\operatorname{ReS}_{2}\right)_{n}-n E(\operatorname{Re})-2 n E(\mathrm{~S})$, are found to be negative, supporting the stability of both structures. Nevertheless, the formation energy per unit cell of S-1 is $\sim 100 \mathrm{meV}$ below that of S-2, implying a better stability for S-1. However, a comparison of this energy difference with the computed exfoliation energies of the layered $\mathrm{ReS}_{2}$ system reveals an interesting property of this structure. The exfoliation energies for $\mathrm{ReS}_{2}$ are computed by using the formula [38] $E_{\text {ex }}(n)=\left[E_{\text {slab }}(n)-E_{\text {bulk }} / m\right] / A$, where $E_{\text {ex }}(n)$ is the exfoliation energy of $n$ layers, $E_{\text {slab }}(n)$ is the energy of 


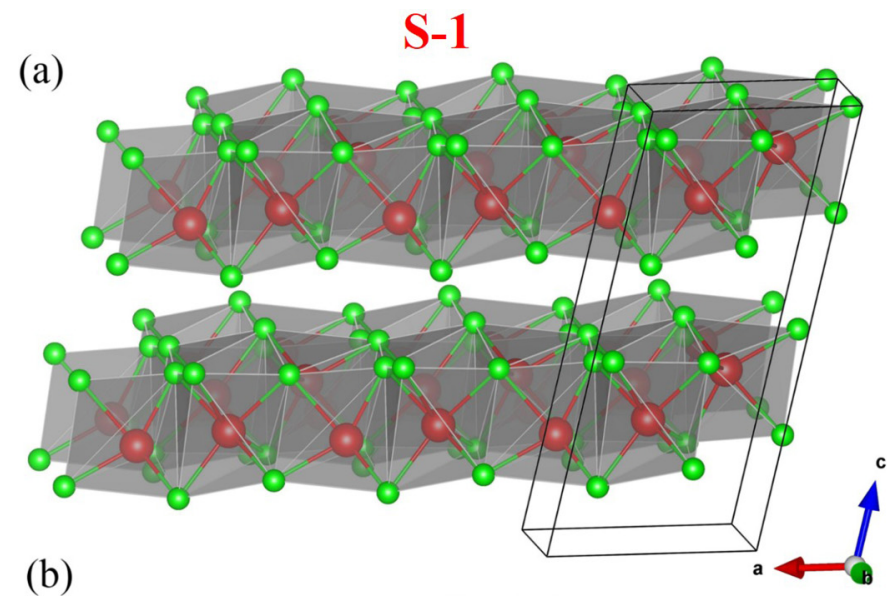

(c)
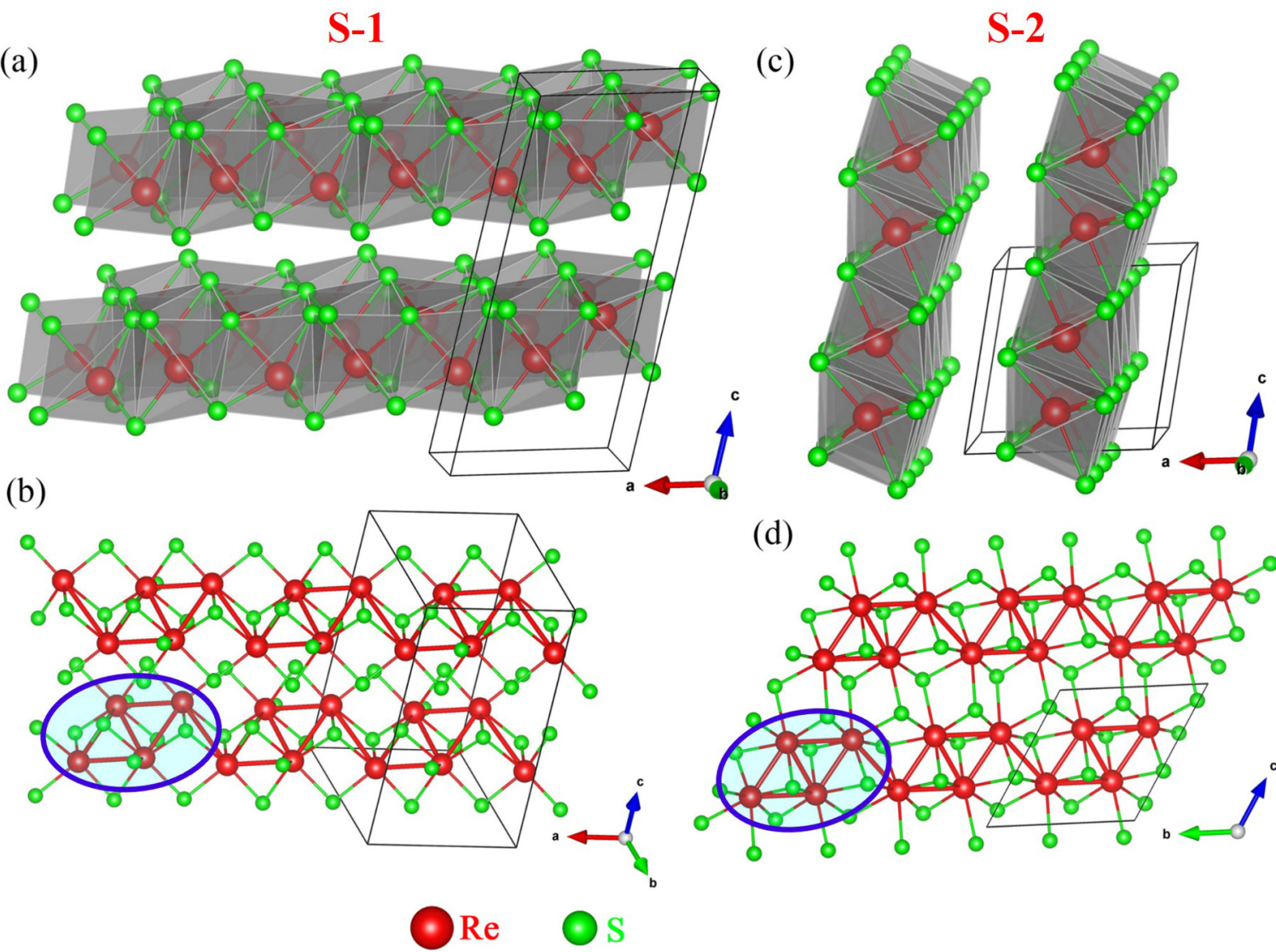

FIG. 1. Polyhedral coordination of octahedra (OCT) and square pyramids (SQP) for (a) S-1 with the corresponding unit cell orientation and (b) the Re4 parallelogram array along the $a$ direction (a typical Re4 parallelogram highlighted within blue ellipse); (c) the OCT polyhedral coordination for S-2 with the respective unit cell orientation and (d) the Re4 parallelogram array along the $b$ direction (highlighted within blue ellipse). Red and green are the colors used for indicating Re and S, respectively.

the unit cell of the $n$-layer slab in vacuum, $E_{\text {bulk }}$ is the energy of the unit cell of the bulk system consisting of $m$ layers, and $A$ is the in-plane area of the bulk unit cell. The exfoliation energy of the monolayer of $\operatorname{ReS}_{2}$ is $\sim 14.7 \mathrm{meV} / \AA^{2}$, and it rises sharply for bilayers and trilayers to $\sim 22$ and $31 \mathrm{meV} / \AA^{2}$, respectively, and soon reaches saturation from five layers onward at $\sim 52 \mathrm{meV} / \AA^{2}$. Such a sharp rise of exfoliation energies explains the frequent use of the ultrasonic or the chemical exfoliation technique for experimental production of few-layered $\mathrm{ReS}_{2}$ flakes [38-40]. Thus, few-layered $\mathrm{ReS}_{2}$ (S-1) systems indicate larger exfoliation energies than other common 2D systems [38]. In addition, within an area of the order of $\AA^{2}$, exfoliation of the few-layered system may lead to a metastable cross-over between S-1 and S-2, as indicated by the difference of their respective formation energies.

As an obvious next step, we have calculated the layer dependence of the electronic structure of monolayer, bilayer, trilayer, and bulk systems of S-1, where the band dispersions and the corresponding partial density of states (PDOS) are plotted in Figs. 2(a)-2(d), respectively. The high-symmetry directions are shown with respect to the corresponding Brillouin zone in Fig. 2(f). Intriguingly, the band dispersions indicate an indirect-to-direct band gap cross-over, with an indirect band gap of $1.46 \mathrm{eV}$ for the monolayer, which gradually reduces due to the energy shift of the valence band (VB) maxima, resulting into a direct band gap of $1.32 \mathrm{eV}$ for the bulk system. Figure 2(e) depicts the in-plane bridging of the face-shared distorted $\operatorname{ReS}_{6}$ OCT chains along the $b$ axis through the two face-shared couplets of $\mathrm{ReS}_{5}$ distorted SQP along the $a$ axis.

Figures 2(g) and 2(h) present the static and time-dependent optical absorbance per unit optical path calculated by using density functional theory (DFT) and time-dependent DFT (TDDFT), respectively, which is compatible with the corresponding band structures. In both cases, with increasing number of layers, there is an increase in the value of the absorbance. The calculated electronic structure and the corresponding optical properties match very well with the experimental results existing in the literature, where with increasing thickness of $\mathrm{ReS}_{2}$ layers, there is an increase in the intensity of luminescence [29].

Presence of metallic clusters and the directionality of its inplane orientation results in highly anisotropic optical response for $\mathrm{ReS}_{2}$, as is also observed experimentally [11]. Figure S1 in the Supplemental Material [37] presents the static anisotropic optical absorbance per unit optical path from the monolayer to bulk system, where all three components of the absorbance are inexorably different.

The band dispersions for S-2 are plotted in Fig. 3, which closely resemble Ref. [30], where the system indicates an indirect band gap all along, except for its trilayer with a direct band gap of $1.28 \mathrm{eV}$. 
(a)

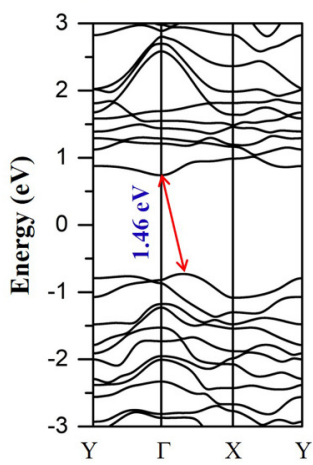

Monolayer (S-1)

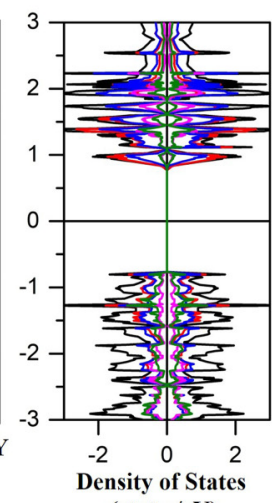

(states/eV)

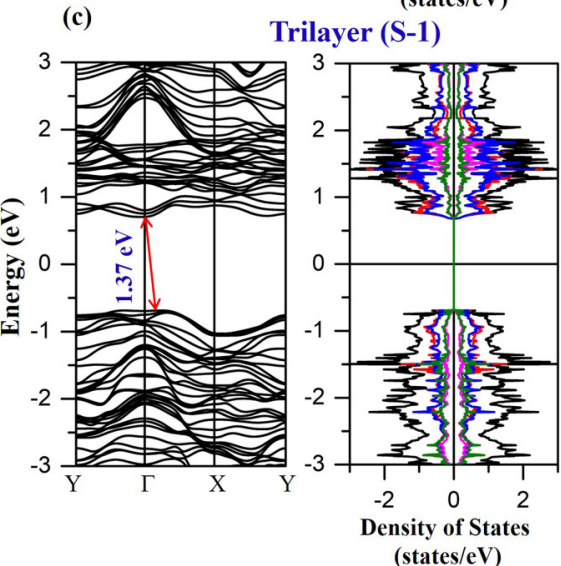

- Total - Re1 - Re8

(Bridge) (Octa) (b)

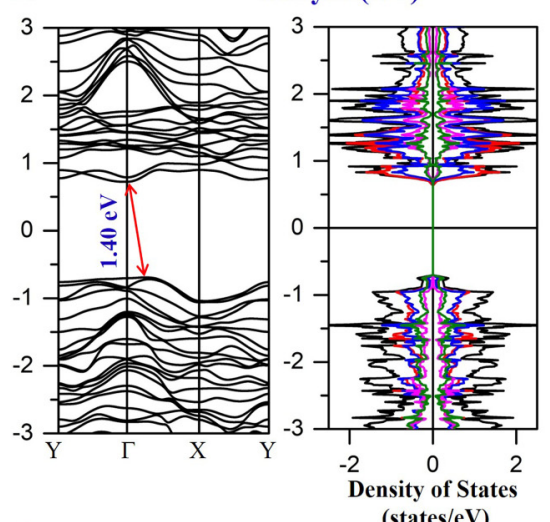

(d)

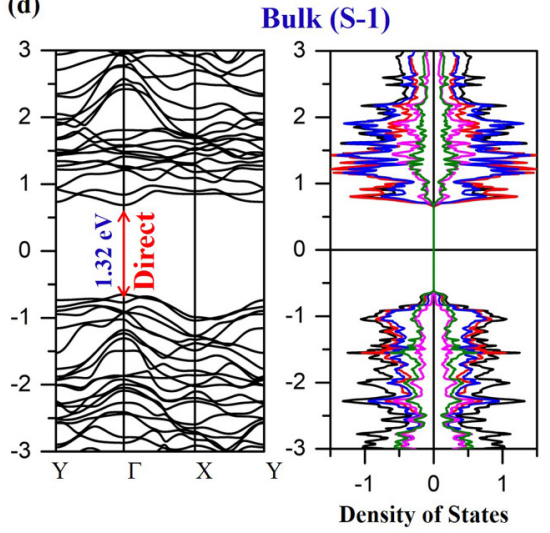

(states/eV)

Bilayer (S-1)

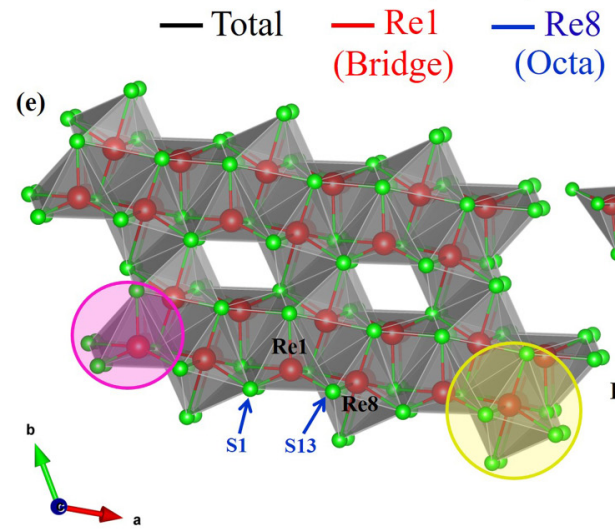

$\underset{\text { (Bridge) }}{\text { S1 }}$

- S13

(Octa)

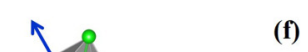

(f)
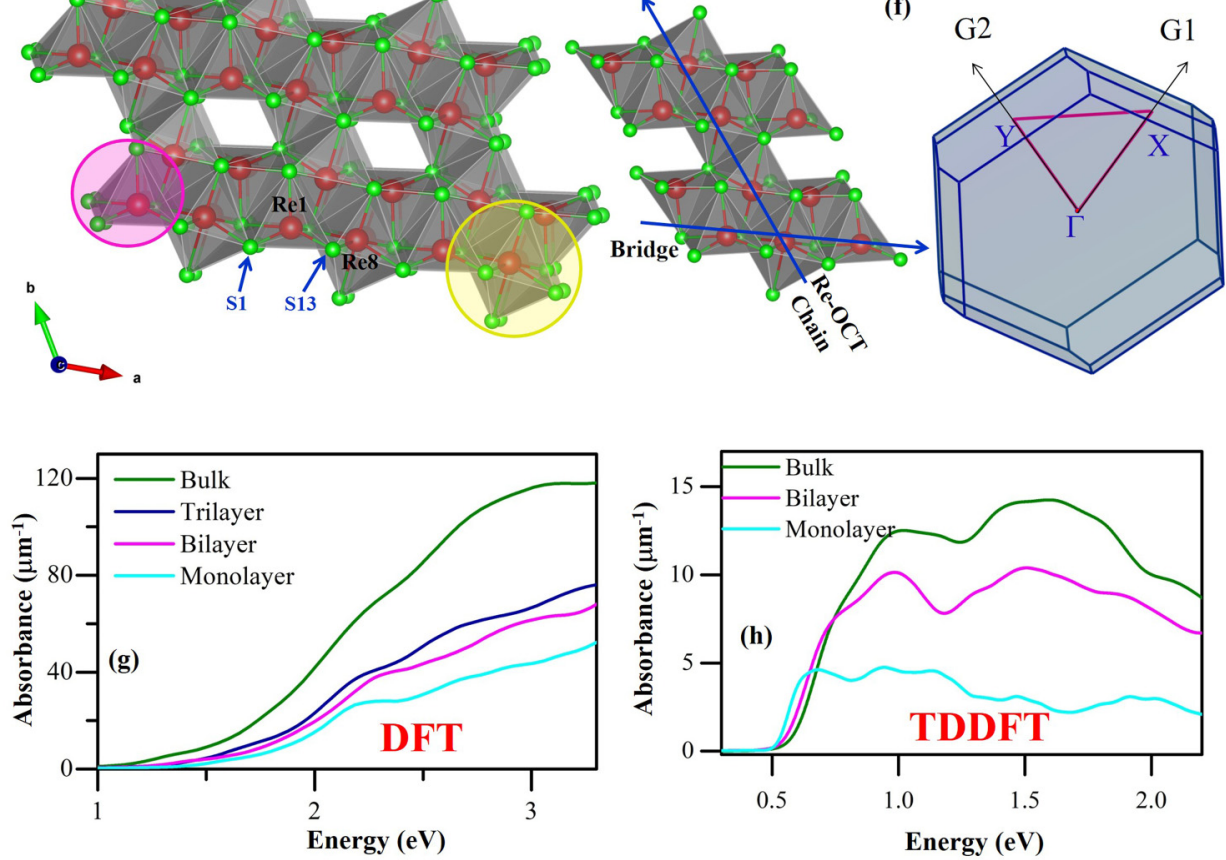

FIG. 2. Band structure and corresponding density of states (DOS) of $\operatorname{ReS}_{2}$ (S-1) (a) monolayer, (b) bilayer, (c) trilayer, and (d) bulk system. (e) Multiple polyhedral coordination of $\mathrm{ReS}_{2}$ in $\mathrm{S}-1$, square pyramidal (SQP) and octahedral (OCT) polyhedra have been marked by using pink and yellow circles, respectively. Right inset shows the one-dimensional (1D) OCT chain and bridging SQP structure (f) corresponding Brillouin zone and the high symmetry directions of the structure. Optical absorbances of different $\operatorname{ReS}_{2}$ systems are plotted after using ( $\mathrm{g}$ ) density functional theory (DFT) and (h) time-dependent DFT (TDDFT) methodologies. 
(a)

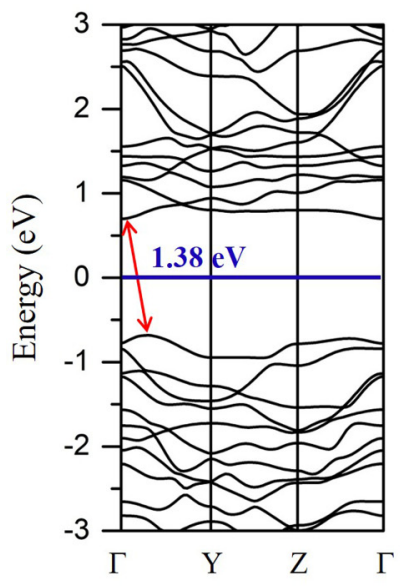

(c)

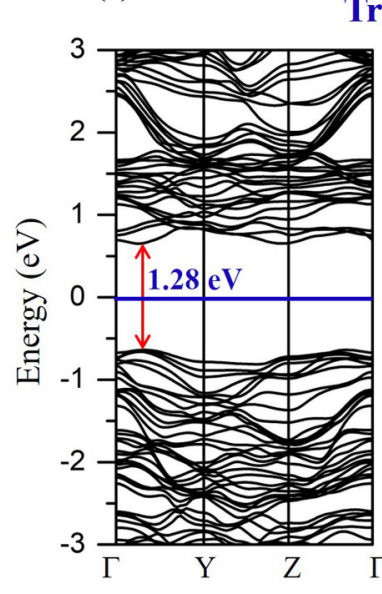

Monolayer (S-2)

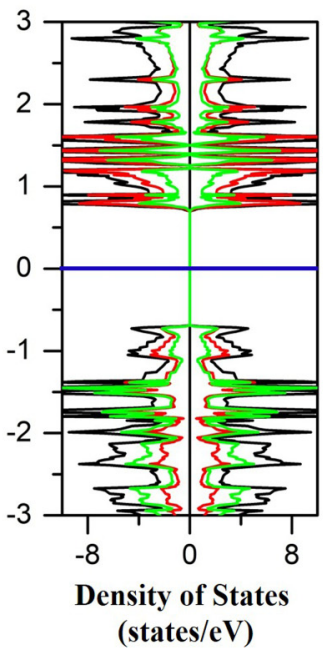

Trilayer (S-2)

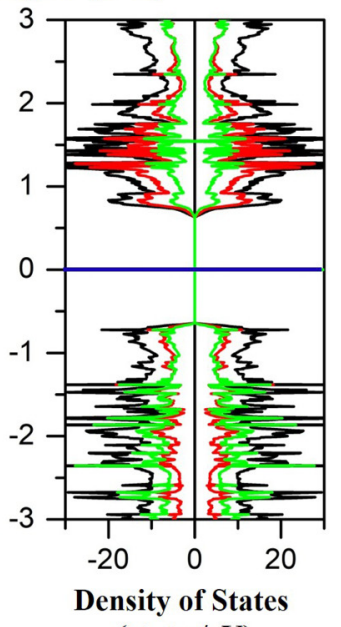

(states/eV) (b)

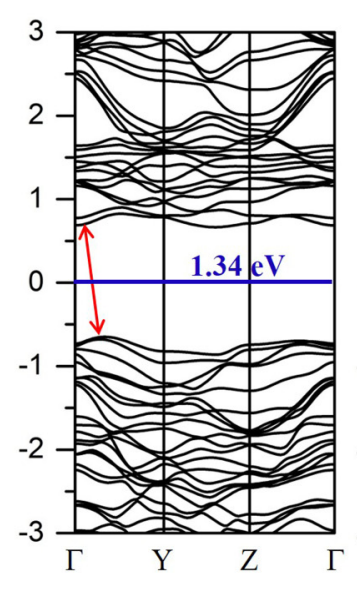

(d)

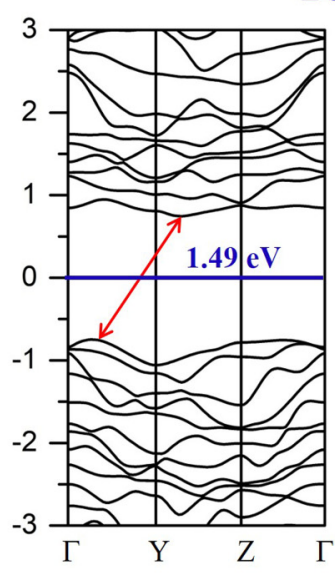

Bulk (S-2)

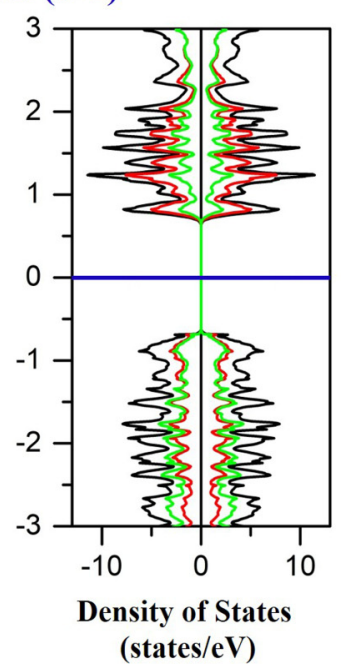

FIG. 3. Layer dependence of band structure and corresponding partial density of states (PDOS) for S-2 of ReS in $1 \mathrm{~T}$ octahedral coordination for (a) monolayer, (b) bilayer, (c) trilayer, and (d) bulk.

Intricate scrutiny of the structural details of S-1 reveals that the $\mathrm{S}$ atoms belonging to OCT and SQP are in different planes, and the inter-planer distances between the $\mathrm{S}$ atoms belonging to the different layers are as short as $\sim 3 \AA$. Thus, S-1 promotes the possibility of inter-planer electronic cross-talking much stronger than the dipolar van der Waals interactions, leading to an increase in the exfoliation energies with the number of layers. Moreover, the distortion of the SQP bridging couplet and the strong hybridization of its center Re atom (Re1) with the constituent $\mathrm{S}$ atoms, which are corner-shared with the OCT chain [S1 and S13, Fig. 2(e)], have a prominent role on the layer dependence of the electronic structure. The states at the top of the VB and the bottom of the conduction band (CB) are mostly populated by the hybridized states of S13 and S1 with Re1 [Figs. 2(a)-2(d)] and are therefore partially responsible for the band gap cross-overs. Additionally, there is a significant hybridization of the terminating $\mathrm{S}$ atoms of a layer with the center Re atoms of OCT and SQP of the next layer via their respective constituent edge $S$ atoms, as will be evident from Figs. 4(a)-4(d) for bulk, monolayer, bilayers, and trilayers of $\mathrm{ReS}_{2}$. This hybridization becomes more prominent with increasing number of layers and may provide another reason behind the indirect-to-direct band gap cross-over from the monolayer to bulk. Thus, both intra- and inter-layer polyhedral Re-S hybridizations are culpable for the layer-dependent electronic response.

$\mathrm{ReS}_{2}$ is also well known to have various possible defects [41], actuating its use in producing solid state lasers [42]. Figure 5 depicts the role of $\mathrm{S}$ vacancies at the (a) OCT, (b) SQP, and (c) both sites, indicating the presence of highly localized mid-gap levels and an associated $n$-type doping, as seen in other TMDCs, too [43].

In the next section, we carry out the experimental investigations on large-area $\mathrm{ReS}_{2}$ samples to endorse our theoretical results. 


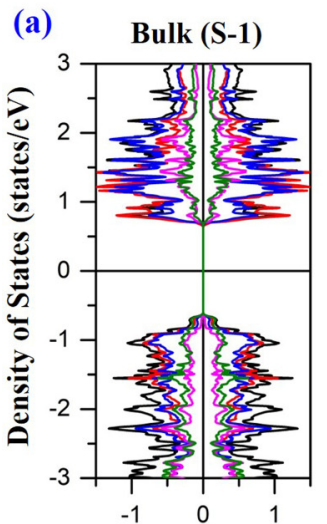

Energy (eV) (b) Monolayer (S-1)

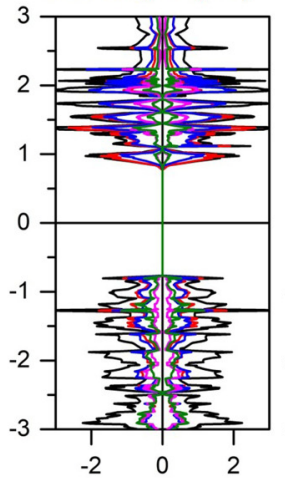

Energy (eV) (c) Bilayer (S-1)

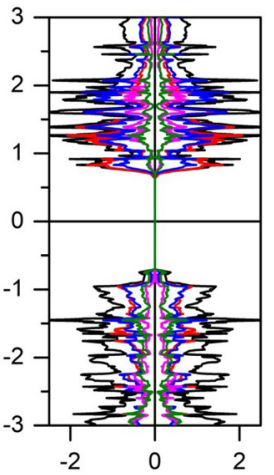

Energy (eV) (d) Trilayer (S-1)

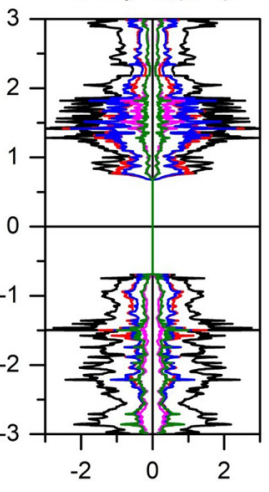

Energy (eV) (e)

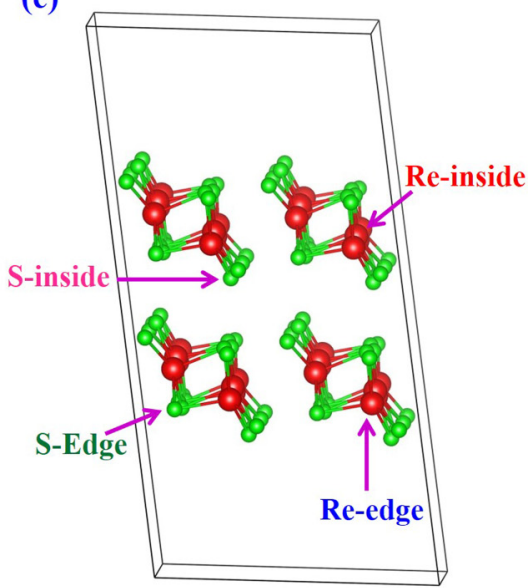

$\longrightarrow$ Total $\longrightarrow \operatorname{Re}($ inside) $\longrightarrow \operatorname{Re}($ edge $) \longrightarrow S($ inside) $\longrightarrow S$ (edge)

FIG. 4. Contribution of edge and inside Re and S atoms in the density of states (DOS) of (a) bulk, (b) monolayer, (c) bilayer, and (d) trilayer. (e) The edge and inside atoms considered in the partial density of states (PDOS).

\section{EXPERIMENTAL ANALYSIS: SYNTHESIS AND CHARACTERIZATION}

With a clearer idea about the structural details, we have looked for experimental validation of the theoretically obtained results, initiating with the synthesis of $\mathrm{ReS}_{2}$ in the proper phase. There are numerous effective methods to synthesize a single-phase multilayered $\mathrm{ReS}_{2}$ system starting from the top-down nonscalable methods like mechanical or liquid exfoliation [39], high-temperature halogen-assisted growth of single crystals [44] to the scalable bottom-up epitaxial growth process like chemical vapor deposition (CVD) [45-47]. Synthesis of $\mathrm{ReS}_{2}$ by direct sulfurization of ammonium perrhenate $\left(\mathrm{NH}_{4} \mathrm{ReO}_{4}\right)$ is known to be an effective and relatively low-temperature process to obtain the largearea films at an ambient pressure [46,48-50]. The details of this synthesis procedure are described in the Supplemental Material [37]. In this paper, single-phase multilayered $\mathrm{ReS}_{2}$ thin films are grown on the $\mathrm{Si} / \mathrm{SiO}_{2}$ wafers at three different growth temperatures, viz., 450,650 , and $750{ }^{\circ} \mathrm{C}$. We designate the films grown at these three different temperatures as F1, F2, and F3, respectively. Unlike the other TMDC systems, $\mathrm{ReS}_{2}$ is well known for its inherent spontaneity toward a nonplanar vertical wall-like or ribbonlike CVD growth, irrespective of the choice of the substrate [48-53]. Several propositions attempt to unravel the reason behind this vertical mode of growth, including the mismatch between the rates of the supply of the precursors to their respective rates of diffusion and the stress-induced curling, originating from its lattice mismatch with the substrate [51]. Ghosal et al. [48] demonstrated the two-step nature of the CVD growth, viz., the initial horizontal growth providing full coverage to the substrate, followed by the successive vertical ones that originate from the out-of-plane protrusions of the OCT $\mathrm{S}$ atoms and the Re-Re metallic bonds. Such vertical growths, although demonstrated for an improved applicability in catalysis, field emission, energy storage, and water purification [48,51], are scarcely validated for their pertinence in the field of excited (a)

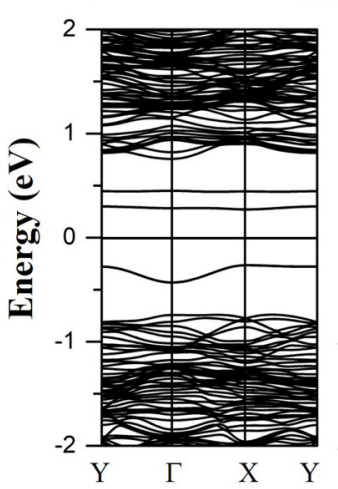

Vacancy at OCT (S-1)

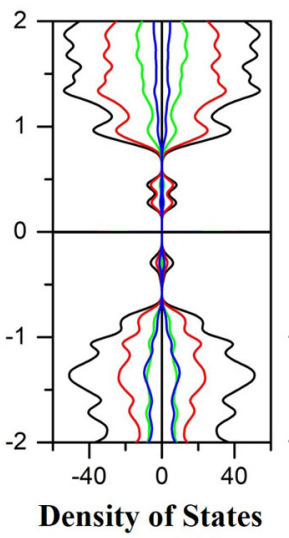

(states/eV) (b)

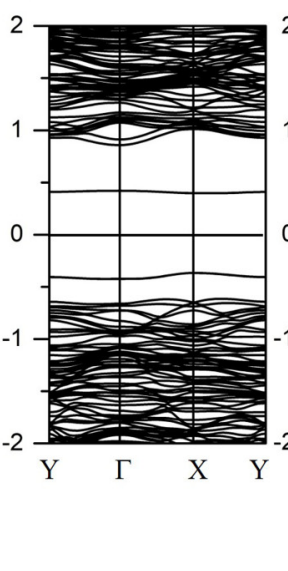

Vacancy at SQP (S-1)

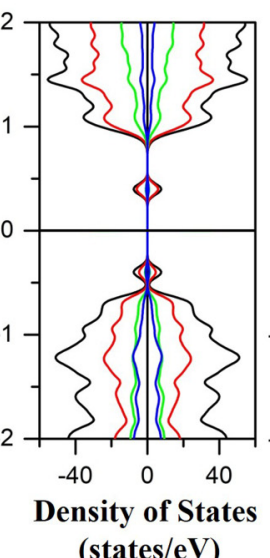

(c)

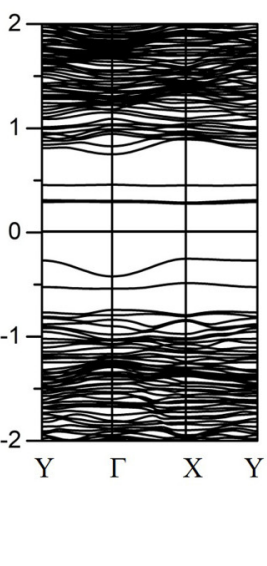

Vacancy at Both (S-1)

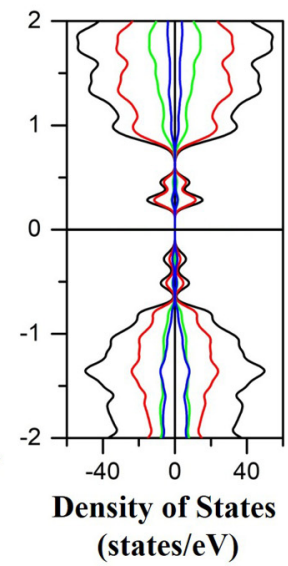

FIG. 5. Band structure and corresponding partial density of states (PDOS) of bulk $\operatorname{ReS}_{2}$ (S-1) with sulfur vacancies at the (a) distorted octahedra (vacancy at OCT), (b) square pyramid (vacancy at SQP), and (c) at both polyhedral places (vacancy at both). 

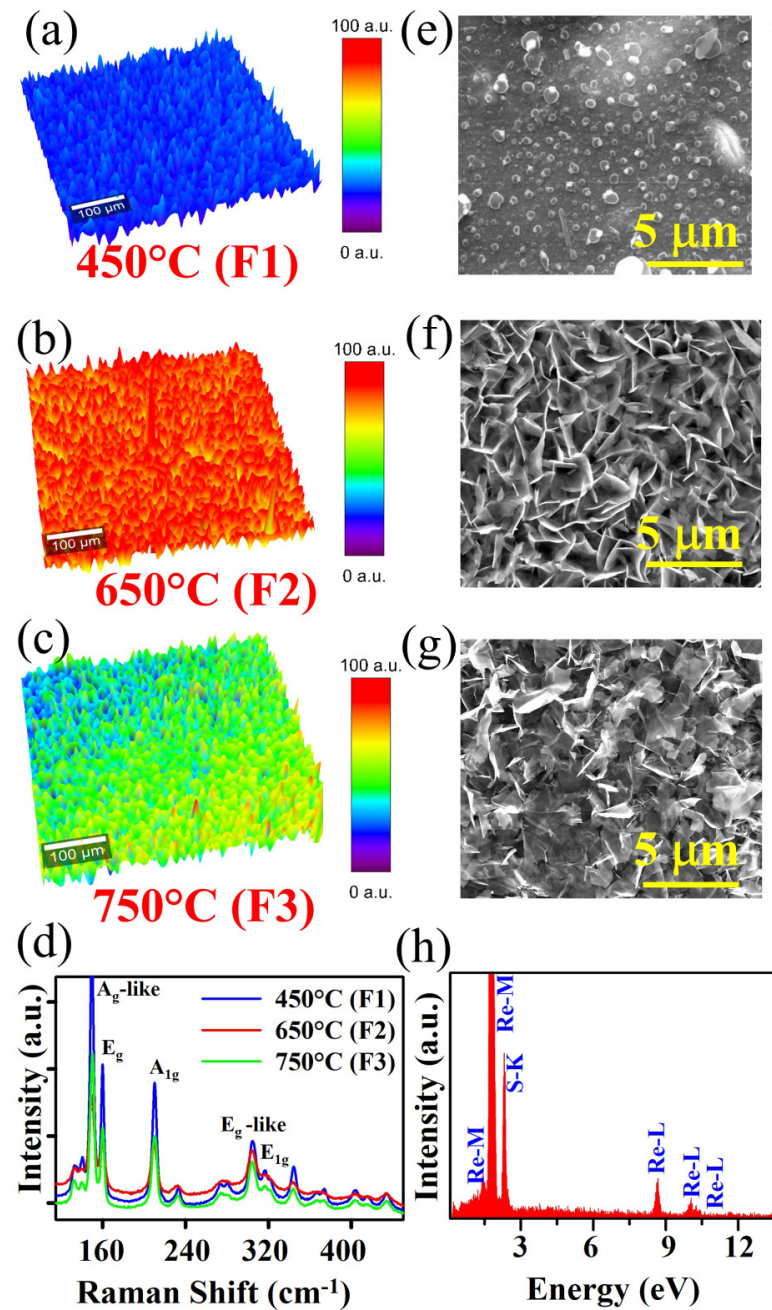

(h)

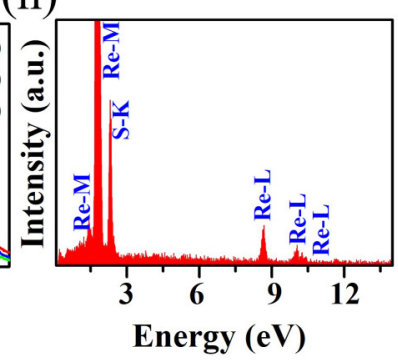

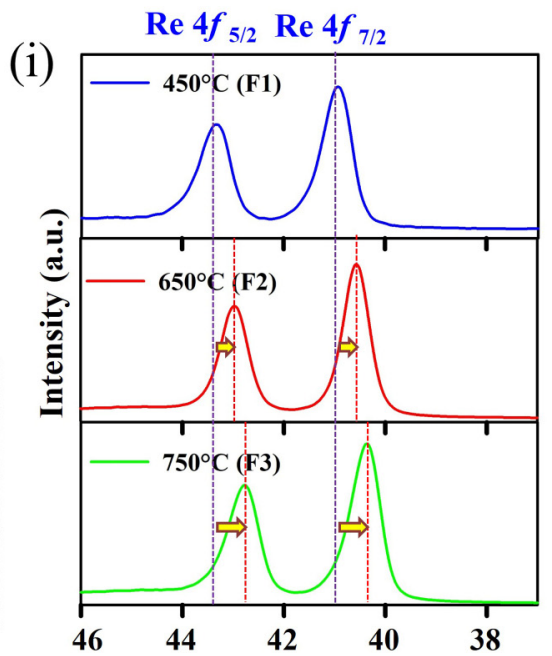

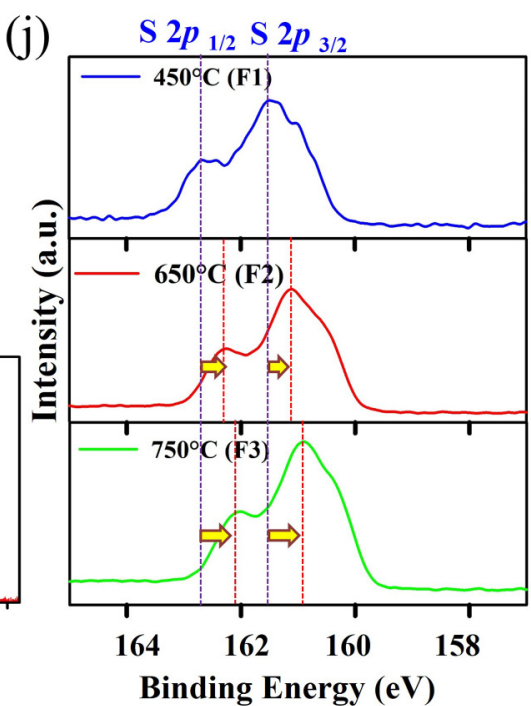

FIG. 6. Three-dimensional (3D) Raman maps of different chemical vapor deposition (CVD) grown films at different growth temperatures: (a) $450{ }^{\circ} \mathrm{C}(\mathrm{F} 1)$, (b) $650{ }^{\circ} \mathrm{C}(\mathrm{F} 2)$, (c) $750{ }^{\circ} \mathrm{C}$ (F3), and their corresponding (d) Raman spectra. Corresponding scanning electron microscopic images of different films for (e) $450{ }^{\circ} \mathrm{C}$ (F1), (f) $650{ }^{\circ} \mathrm{C}(\mathrm{F} 2)$, (g) $750{ }^{\circ} \mathrm{C}$ (F3); (h) energy-dispersive x-ray (EDX) elemental map of ReS 2 $\left(650^{\circ} \mathrm{C}\right)$; XPS spectra of (i) Re- $4 f$ and (j) S-2 $p$ electrons in different samples (F1-F3).

state photo-response and opto-electronics. In the current experimental part of this paper, we have emphasized over such applications.

Figure 6 presents the micro-Raman (MR), energy dispersive $\mathrm{X}$-ray (EDX) analysis and the $\mathrm{X}$-ray photoelectron spectra (XPS) as a function of the different growth temperatures. As supporting evidence toward a uniform large-area deposition of the proper crystalline phase, the MR spectra for the feasible Raman active modes were reproduced from the average of several Raman mappings over an area of $500 \times 500 \mu \mathrm{m}$ at various places of each film with a laser of wavelength $532 \mathrm{~nm}$ and a spot size of $\sim 20 \mu \mathrm{m}$. For $\operatorname{ReS}_{2}$, unlike the other TMDC systems, the lack of symmetry, intra-mode couplings and the interactions with acoustic modes incite 36 vibrational modes altogether. Keeping apart the 15 infrared active and three zero-frequency modes, the remaining 18 Raman active ones constitute the $A_{g}$-like and $E_{g}$-like vibrational modes, originating from the out-of-plane and in-plane vibrations of the Re atoms, respectively [54]. Figures 6(a)-6(c) depict the Raman intensity maps with the associated color scales, after considering the most intense $A_{g}$-like $\left(149 \mathrm{~cm}^{-1}\right)$ peak, having much less sensitivity toward polarization. The Raman maps evinced that $\mathrm{F} 2\left(650^{\circ} \mathrm{C}\right)$ has the most uniform and intense Raman spectra, as is also manifested in the MR spectral plots for F1-F3 in Fig. 6(d). The peak positions for the possible Raman modes, corresponding to Fig. 6(d), are presented in Table I for F1-F3, indicating negligible frequency shifts. The $A_{g^{-}}$and $E_{g}$-like peaks occur at $\sim 149$ and $304 \mathrm{~cm}^{-1}$, respectively. Higher energy $E_{g}$-like modes and other peaks originate from the out-of-plane vibrations of the $S$ atoms. In addition to those, two prominent modes $E_{g}$ and $A_{1 g}$ are observed at 160 and $210 \mathrm{~cm}^{-1}$, respectively [50,55]. Figures $6(\mathrm{e})-6(\mathrm{~g})$ represent the scanning electron microscopic images for all three films F1-F3. With increasing temperature, the 2D film F1 [Fig. 6(e)] evolves to obtain a uniformity for the wall-like growth at F2 [Fig. 6(f)], which is disrupted with the increase in growth temperature, as in F3 [Fig. 6(g)]. The corresponding elemental map of F2 is presented in Fig. 6(h), and Table I presents the percentage compositions of Re and $\mathrm{S}$ for $\mathrm{F} 1-\mathrm{F} 3$, with a clear indication of their 1:2 ratios for F2. Therefore, 
TABLE I. Raman peak positions and EDX elemental map of different samples.

\begin{tabular}{|c|c|c|c|c|c|c|c|}
\hline \multirow{2}{*}{$\begin{array}{l}\text { Growth temperature } \\
\text { (sample name) }\end{array}$} & \multicolumn{5}{|c|}{ Raman peak position } & \multicolumn{2}{|c|}{ EDX of elements } \\
\hline & $A_{g}\left(\mathrm{~cm}^{-1}\right)$ & $E_{g}\left(\mathrm{~cm}^{-1}\right)$ & $A_{1 g}\left(\mathrm{~cm}^{-1}\right)$ & $E_{g}$-like $\left(\mathrm{cm}^{-1}\right)$ & $E_{1 g}\left(\mathrm{~cm}^{-1}\right)$ & S (at. \%) & $\operatorname{Re}($ at. $\%)$ \\
\hline $450^{\circ} \mathrm{C}(\mathrm{F} 1)$ & 149.7 & 160.0 & 210 & 304.7 & 316.8 & 64.0 & 36.0 \\
\hline $550{ }^{\circ} \mathrm{C}(\mathrm{F} 2)$ & 149.7 & 160.0 & 209.5 & 304.5 & 316.6 & 67.1 & 32.9 \\
\hline $650{ }^{\circ} \mathrm{C}(\mathrm{F} 3)$ & 150.2 & 160.26 & 210.5 & 304.4 & 316.8 & 67.5 & 32.5 \\
\hline
\end{tabular}

F2 turns out to be the most optimally grown film, in terms of the perfect stoichiometry of $\mathrm{ReS}_{2}$. To obtain a correct compositional confirmation and the corresponding valence configuration, the binding energy shifts of the core level electrons are measured with high-resolution XPS with hard $\mathrm{x}$ rays in an energy range of $2-15 \mathrm{KeV}$. The XPS spectra for the Re- $4 f$ and S- $2 p$ peaks are presented in Figs. 6(i) and 6(j), respectively, which connotes the shift of these peaks toward the lower binding energy as a function of the growth temperature from F1 to F3. This shift for higher temperature growths may be associated with the presence of defects, inducing highly localized mid-gap states [56,57]. For the optimized sample $\mathrm{F} 2$, whereas the $\operatorname{Re}-4 f_{5 / 2}$ and $4 f_{7 / 2}$ peaks ensued at 42.99 and $40.55 \mathrm{eV}$, the $\mathrm{S}-2 p_{1 / 2}$ and $2 p_{3 / 2}$ peaks emanate at 160.3 and $161.1 \mathrm{eV}$, respectively. The peak positions for F1-F3 are conferred in Table II and compared with the single-crystal $\mathrm{ReS}_{2}$ data [44]. The general observation for the CVD grown films points out the lower binding energies for the characteristic core-level peaks in comparison with the single-crystalline samples, as was also ascertained in earlier studies [44,46].

Figures 7(a)-7(c) present the thickness profile of F1-F3, as measured after scanning the surfaces by an optical profilometer by using a highly focused white light beam. The uniformity of the area coverage in the samples is quite evident from the corresponding color plots. The corresponding height profiles are depicted in Figs. 7(d)-7(f) for all three systems F1-F3. While analyzing the height profile, we emphasize the two-step growth processes of $\mathrm{ReS}_{2}$. Up to $450{ }^{\circ} \mathrm{C}$, there is only horizontal growth, having a thickness of $\sim 434 \mathrm{~nm}$. With increase of growth temperature, the vertical growth initiates. The height profiles of samples F2 and F3, conveying the total thickness, may appear misleading due to their additional vertical growth on top of the horizontal film. Therefore, for all three systems, the thickness of the horizontal crystallites covering the film can be taken as a few hundred nm, ascertaining them to be in the bulk regime. However, this thickness profile does not convey anything about the horizontal size of the crystallites. For more in-depth investigation of the atomic-scale

TABLE II. XPS binding energies of the Re- $4 f$ and S- $2 p$ electrons and the thickness of different systems.

\begin{tabular}{|c|c|c|c|c|c|}
\hline \multirow[b]{2}{*}{ Samples } & \multicolumn{4}{|c|}{ XPS binding energy } & \multirow[b]{2}{*}{ Thickness (nm) } \\
\hline & $\operatorname{Re}-4 f_{5 / 2}$ & $\operatorname{Re}-4 f_{7 / 2}$ & $\mathrm{~S}-2 p_{1 / 2}$ & $\mathrm{~S}-2 p_{3 / 2}$ & \\
\hline $450^{\circ} \mathrm{C}(\mathrm{F} 1)$ & 43.4 & 41.0 & 162.7 & 161.5 & 434 \\
\hline $650^{\circ} \mathrm{C}(\mathrm{F} 2)$ & 43.0 & 40.6 & 162.3 & 161.1 & 445 \\
\hline $750^{\circ} \mathrm{C}(\mathrm{F} 3)$ & 42.8 & 40.4 & 162.1 & 160.9 & 441 \\
\hline Ref. [44] & 44.58 & 42.18 & 162.78 & 161.74 & - \\
\hline
\end{tabular}

structural properties, we have carried out the cross-sectional transmission electron microscopy (TEM) on the $\mathrm{ReS}_{2} / \mathrm{SiO}_{2} / \mathrm{Si}$ interfaces for the sample $\mathrm{F} 2$, and the outcome is plotted in Figs. $7(\mathrm{~g})-7(\mathrm{j})$. Figure $7(\mathrm{~g})$ clearly represents the growth of the $\mathrm{ReS}_{2}$ films on $\mathrm{Si} / \mathrm{SiO}_{2}$ substrate, having an uneven interfacial morphology due to the diffusion of $\mathrm{ReS}_{2}$ within $\mathrm{SiO}_{2}$. As is also observed in the prior studies [48], there are two different types of regions for these thin films, viz., the top vertical growth and a flat coverage of $\mathrm{ReS}_{2}$ beneath the vertical structure. Figure 7(h) depicts the fast Fourier transform image, which clearly conveys the sixfold symmetry of the pattern, confirming the high crystalline nature of the films. When the electron beam is focused on the flat area, the highresolution TEM image clearly shows the Re4 parallelogram cluster arrays covering the entire region having an average distance of $5.7 \AA$ between the two arrays, as obtained from Fig. 7(i). This distance closely resembles the same value as that obtained from the theoretical calculations $(5.4 \AA)$ for the structure $S-1$. The sides of the parallelogram range from 2.76 to $2.84 \AA$ from the measurements, which are comparable with the theoretically obtained lengths of $2.80-2.85 \AA$. The corresponding distance between the parallelogram arrays for $\mathrm{S}-2$ is $5.1 \AA$ with the sides varying from 2.3 to $2.4 \AA$. Albeit the dimensions related to the Re4-parallelogram arrays are comparable for both structures, with $\mathrm{S}-1$ being closer to the experiment, there are more details to be explored from the TEM micrograph. While the beam is focused on the vertical growth pattern, as in Fig. 7(j), the obtained fringes imply an inter-layer distance of $2.4 \AA$, corresponding to the [202] planes of the theoretically predicted structure S-1. Thus, the structural details, as obtained from the experimental large-area growths of $\mathrm{ReS}_{2}$, indicate our experimental structure to be closer to S-1.

We may also mention in passing two important aspects of the as-grown films. First, the theoretically obtained higher range of exfoliation energies for the structure S-1 was supported by the unsuccessful attempts at mechanical exfoliation from the as-grown CVD films, leading to a peeling of the entire film from the substrate. Second, the XPS results imply that the as-grown films, especially grown at higher temperatures, have many defects in comparison with single crystals.

The structural analysis of the as-grown CVD films indicated the evidence of the presence of the structure S-1 at a local level. However, the complicated process of CVD growth, initiating in a planar geometry and followed by a cross-over to a vertical one, results in a complex composite film material. There is further need of more sophisticated and intricate analysis at both microscopic and macroscopic level to unambiguously unravel the structure-properties correlations, which is beyond the scope of this paper. 


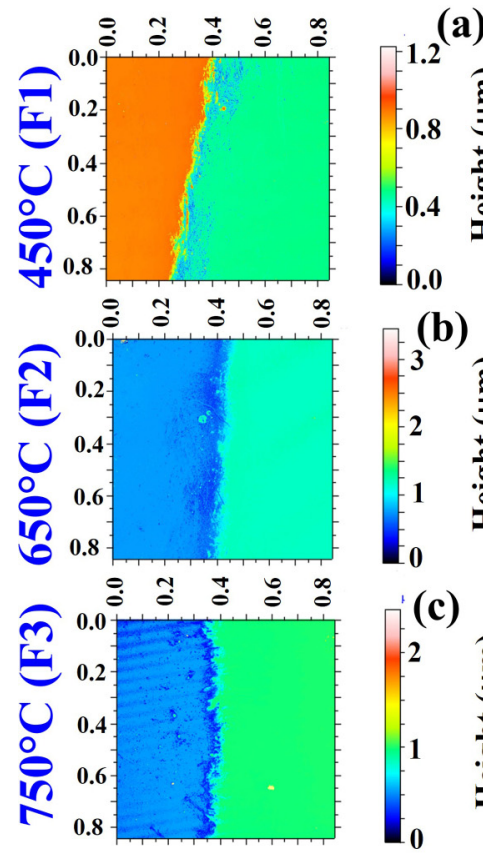

(a)

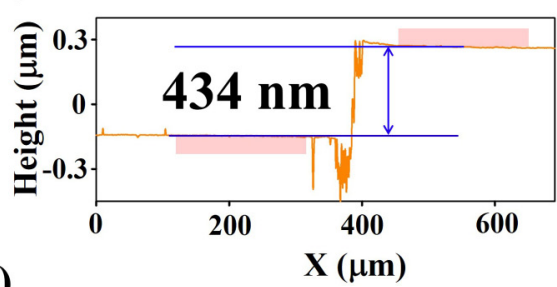

)
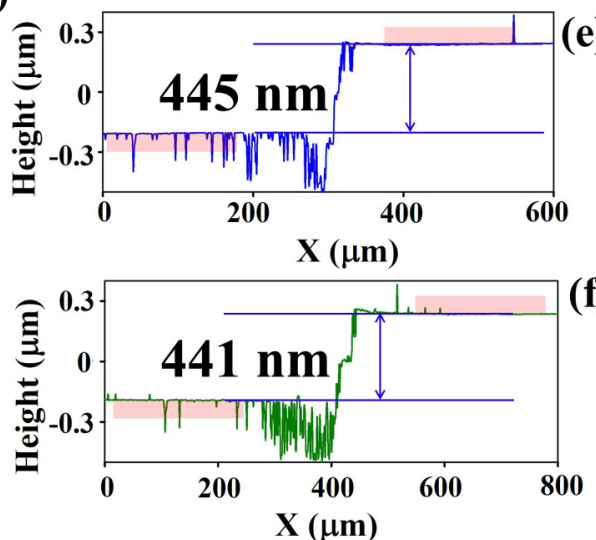

(d)

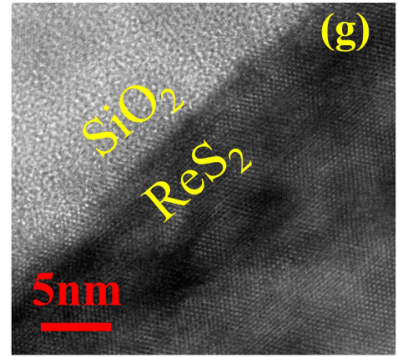

(f)

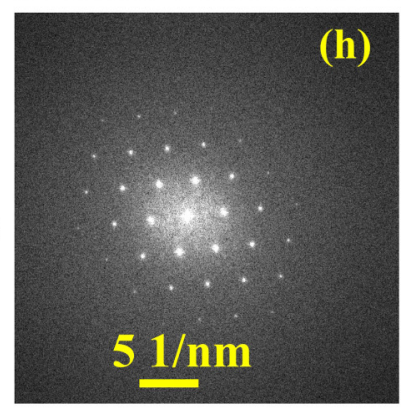

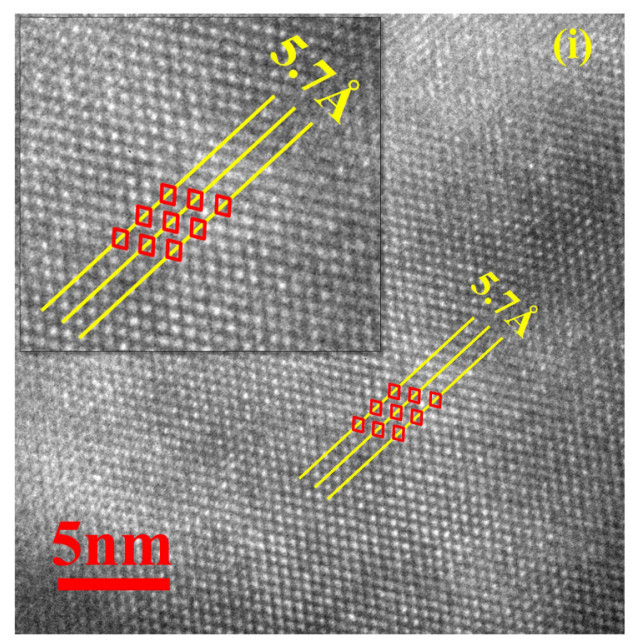

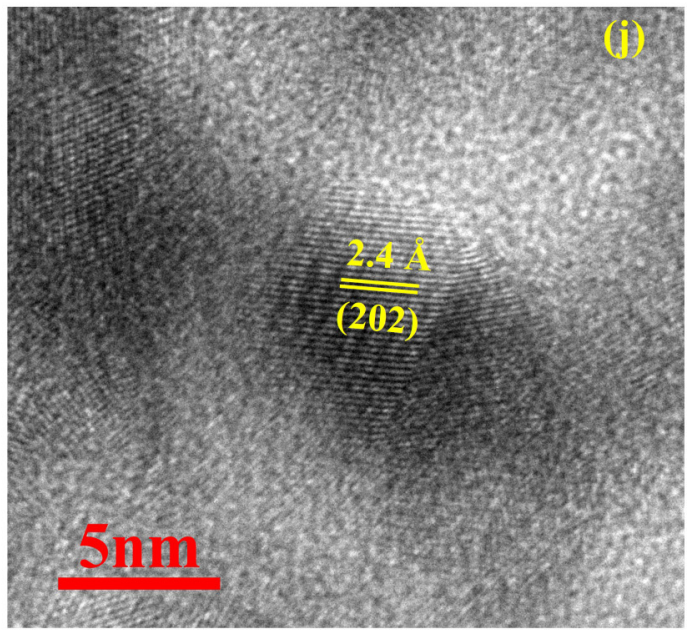

FIG. 7. Color map of thickness of $\mathrm{ReS}_{2}$ samples using an optical profilometer for growth temperatures (a) $450{ }^{\circ} \mathrm{C}(\mathrm{F} 1)$, (b) $650{ }^{\circ} \mathrm{C}(\mathrm{F} 2)$,

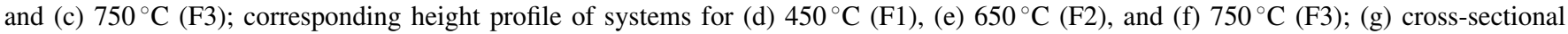
transmission electron microscopy (TEM) images of $\mathrm{ReS}_{2}$ on $\mathrm{SiO}_{2}$ (F2), (g) fast Fourier transform image of ReS image of planar $\mathrm{ReS}_{2}$ depicting the Re4 parallelogram, (j) high-resolution TEM image of vertical growth of ReS $\mathrm{S}_{2}$ showing lattice fringes corresponding to the [202] plane of S-1 (see text).

In the next section, we perform the static and dynamic optical measurements on these systems to highlight the applicability of these vertically grown systems in the field of optics and opto-electronics.

\section{STATIC AND DYNAMIC OPTICAL RESPONSE OF THE CVD FILMS}

Both steady state PL and excited state transient absorption (TA) measurements were performed on the samples F1-F3 to obtain an assessment about their potential optical attributes. PL was measured with a laser of wavelength $532 \mathrm{~nm}$, a spot size of $20 \mu \mathrm{m}$ diameter, and the maximum laser power of $1.6 \mathrm{~mW}$. The main feature of the PL spectra of $\mathrm{ReS}_{2}$ is its significantly low quantum yields for the bulk samples F1-F3, resembling the prior studies. This outcome can be explained from our derived band structure. The difference between the indirect band gap for few-layered samples and the direct band gap for bulk system is $\sim 0.1 \mathrm{eV}$, as seen from Fig. 2. Such a small difference in the band gap allowed the probabilities of the other transitions and thus broadens the peak and produces different shapes for the line profiles. In Fig. 8(a), for F1, there is presence of both $\mathrm{A}$ and $\mathrm{B}$ excitons with the intensity of the latter being higher, as is also seen in many prior studies [11]. The higher B exciton is attributed to the presence of a rapid nonradiative relaxation associated with the presence of sulfur vacancy (SV) defects [11] or the lower oscillator strength of exciton A than exciton B. As can also be seen from the Fig. 5, the SV defects create highly localized shallow traps within the band gap of $\mathrm{ReS}_{2}$, providing a suitable means for relaxation 


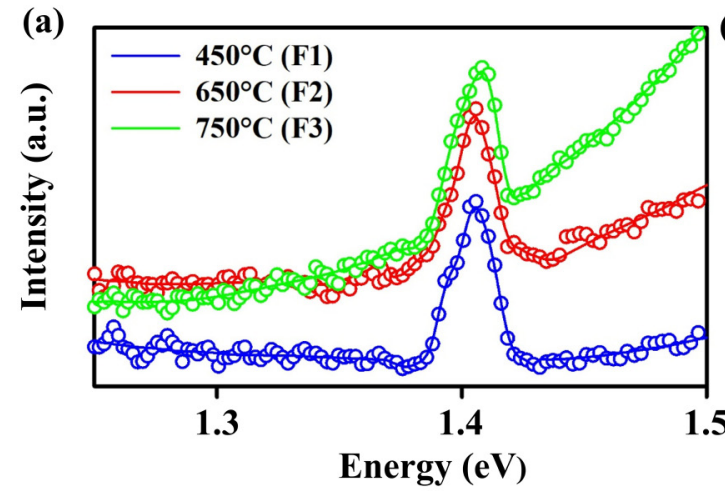

(b)
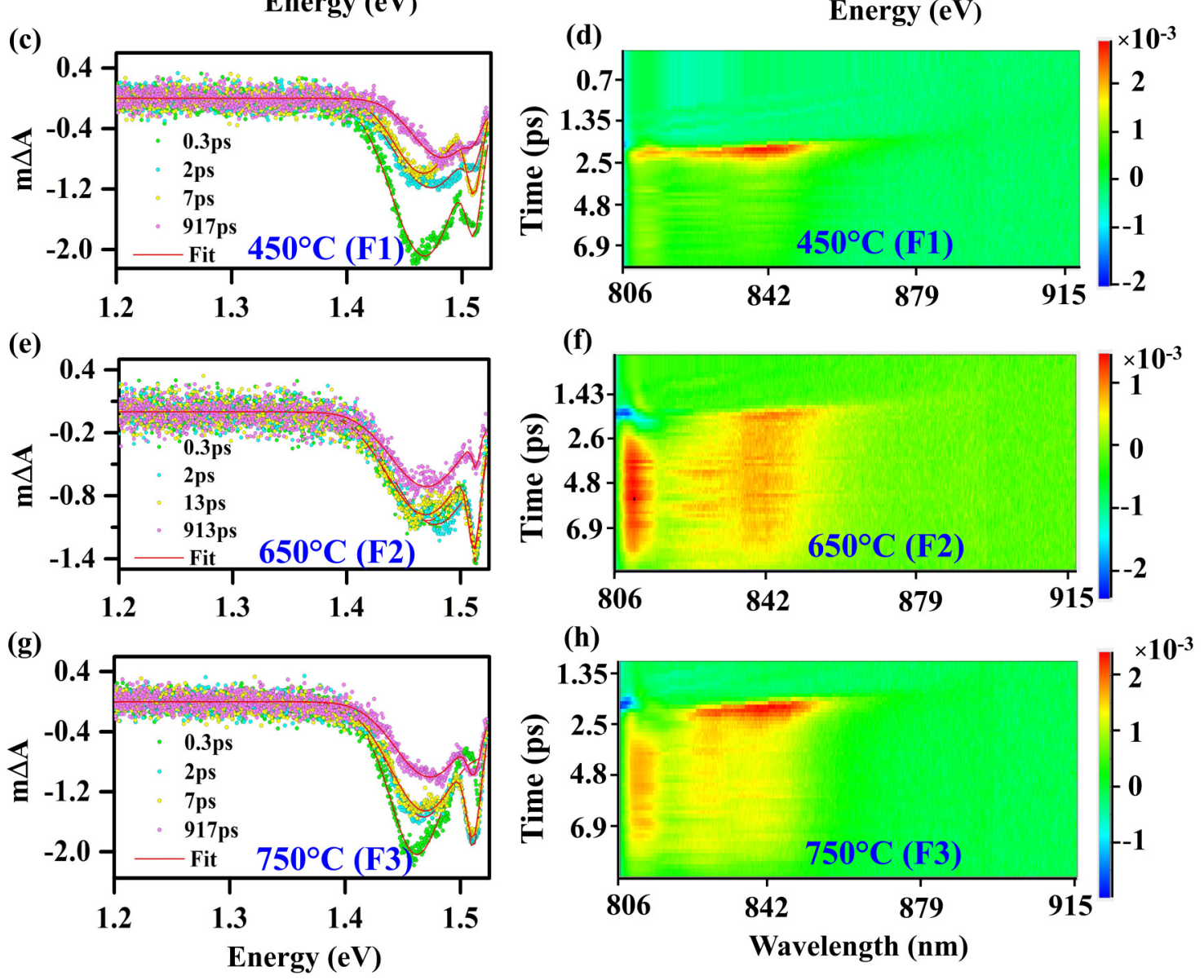

FIG. 8. Room temperature photoluminescence (PL) spectra of F1, F2, and F3 $\left(650^{\circ} \mathrm{C}\right)$ (a) at lower energy and (b) higher energy range. Kinetics for exciton decay for (c) $\operatorname{ReS}_{2}\left(450{ }^{\circ} \mathrm{C}, \mathrm{F} 1\right)$, (e) $\operatorname{ReS}_{2}\left(650^{\circ} \mathrm{C}, \mathrm{F} 2\right)$, and $(\mathrm{g}) \operatorname{ReS}_{2}\left(750^{\circ} \mathrm{C}, \mathrm{F} 3\right)$. The contour plots of transient absorption (TA) measurements for (d) $\operatorname{ReS}_{2}\left(450{ }^{\circ} \mathrm{C}, \mathrm{F} 1\right)$, (f) $\operatorname{ReS}_{2}\left(650^{\circ} \mathrm{C}, \mathrm{F} 2\right)$, and (h) $\operatorname{ReS}_{2}\left(750{ }^{\circ} \mathrm{C}, \mathrm{F} 3\right)$.

of the conduction electrons. For the other two samples F2 and F3, only a single exciton (B) signal is present. With increasing vertical growth, there will be a simultaneous increase in the defect densities, rendering a suppression of the A exciton. The PL peak of the $\mathrm{B}$ exciton is at $\sim 1.40 \mathrm{eV}$ for $\mathrm{F} 1$, and with an increase in the growth temperature, there is a small blueshift in the peak positions, indicating stronger electronic couplings for high temperature samples. Additionally, the smaller exciton binding energy in $\operatorname{ReS}_{2}$, as seen by Aslan et al. [11], can be reproduced from the difference of the theoretically calculated optical band gap $(1.32 \mathrm{eV})$ and the experimental exciton positions $(1.40 \mathrm{eV})$. In addition to the lower energy exciton peaks, the samples display higher energy peaks from
1.8 to $2.0 \mathrm{eV}$, as depicted in Fig. 8(b), the presence of which can be explained from the PDOS figure of the bulk system [Fig. 2(d)]. The VB and CB DOS peaks at $\sim-1$ and $1 \mathrm{eV}$, respectively, corresponding to the S-hybridized OCT and SQP $\mathrm{Re}-5 d$ states, are responsible for the origin of the higher energy peaks.

The evolution of the excited state dynamics for the films F1-F3 was probed with the picosecond resolved TA spectroscopy, where the samples were excited with a beyond band gap pump beam of energy $3.1 \mathrm{eV}$, and the resulting excitations were explored with a probe beam with energy ranging from 1.76 to $2.6 \mathrm{eV}$. Figures $8(\mathrm{c}), 8(\mathrm{e})$, and $8(\mathrm{~g})$ depict the spectral plots representing the change in the absorbance 

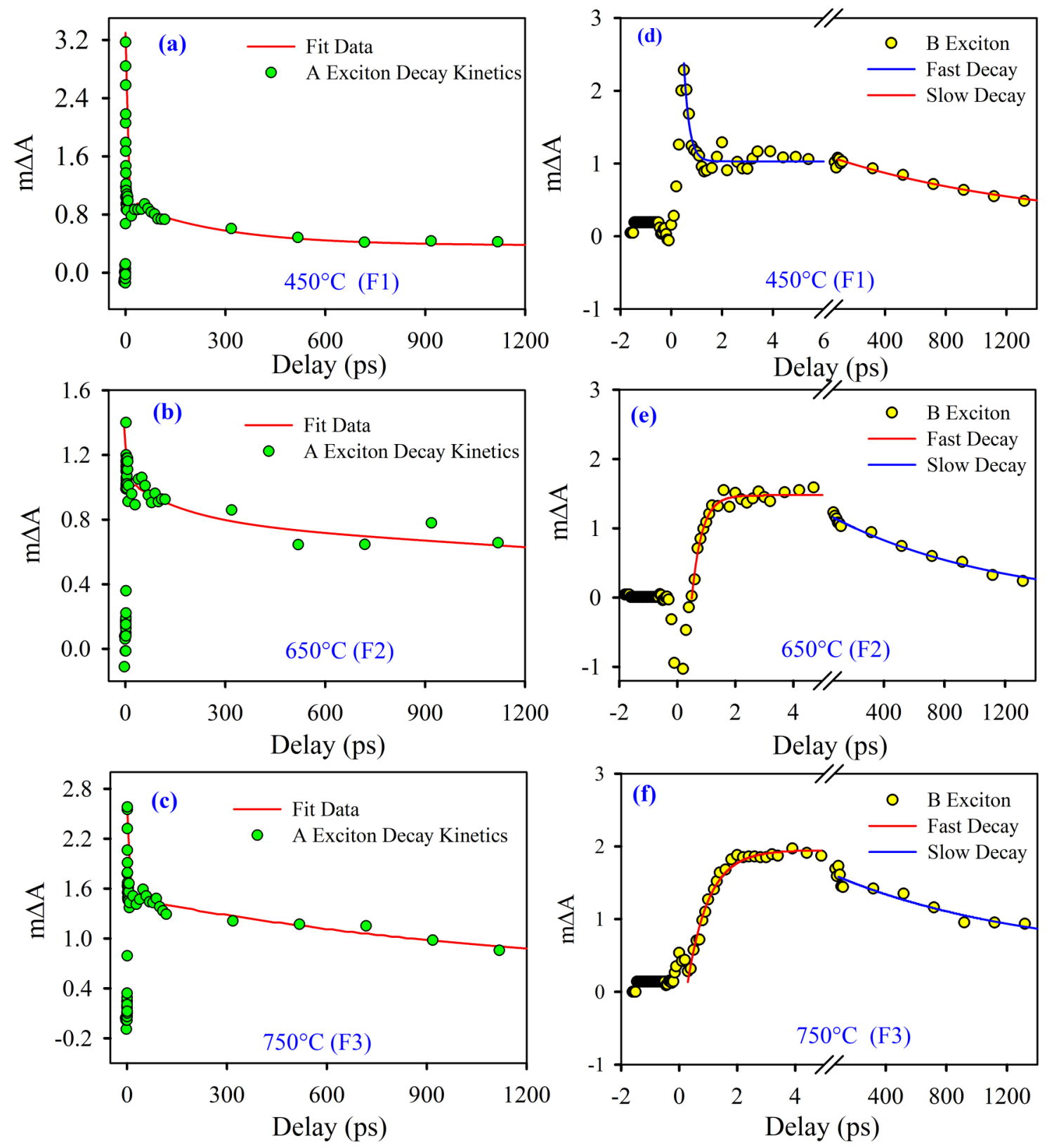

FIG. 9. Kinetics for A-exciton decay and trap state filling for (a) F1, (b) F2, and (c) F3. Exciton signals are seen to be alive even after 1200 ps. Kinetics for B-exciton slow decay, fast decay, and trap state filling at for (d) F1, (e) F2, and (f) F3. Exciton signals are seen to be alive even after 1200 ps.

$m \Delta A=A_{\text {excited }}-A_{\text {ground }}$ between the excited and ground states as a function of energy. The corresponding kinetics of the excitons are presented in the contour plots in Figs. 8(d), $8(\mathrm{f})$, and $8(\mathrm{~h})$, respectively, where the delay time between the pump and probe beams are plotted as a function of the wavelength, with the color codes in $m \Delta A$ presented as a side bar. All three samples have displayed the clear presence of both $\mathrm{A}$ and $\mathrm{B}$ excitons at $\sim 1.47$ and $1.52 \mathrm{eV}$, respectively, from the TA optical data of Figs. 8(c), 8(e), and $8(\mathrm{~g})$. For $\mathrm{F} 1$ and $\mathrm{F} 3$, exciton $\mathrm{A}$ is more intense than $\mathrm{B}$, whereas for $\mathrm{F} 2$, exciton $\mathrm{B}$ remains more prominent. A significant bleach-cum-state filling is observed for all three systems, with the indication of F2 producing the most uniform contour plot. All these figures conveyed two extremely intriguing excited state features for the CVD films. First, except for the planar sample F1, both vertical growths F2 and F3 show a significantly large lifetime of excitons, with an indication of survival even $>917$ ps. The contour plots indicate significant bleaches after a delay of more than 6900 fs. The decay time and the filling up of the trap states corresponding to both exciton signals are plotted in Figs. 9(a)9(d), indicating persistent excitons even after 1200 ps. Such high lifetimes of excitons can be explained from the theoretical implications of Fig. 5, where the extremely localized $\mathrm{SV}$-induced mid-gap states act as electron traps, imparting a longer lifetime for the excitons. Secondly, for F1 and F3, with increasing delay time, there is a blueshift in the energy of the excitons, known as the Stark shift. The occurrence of 

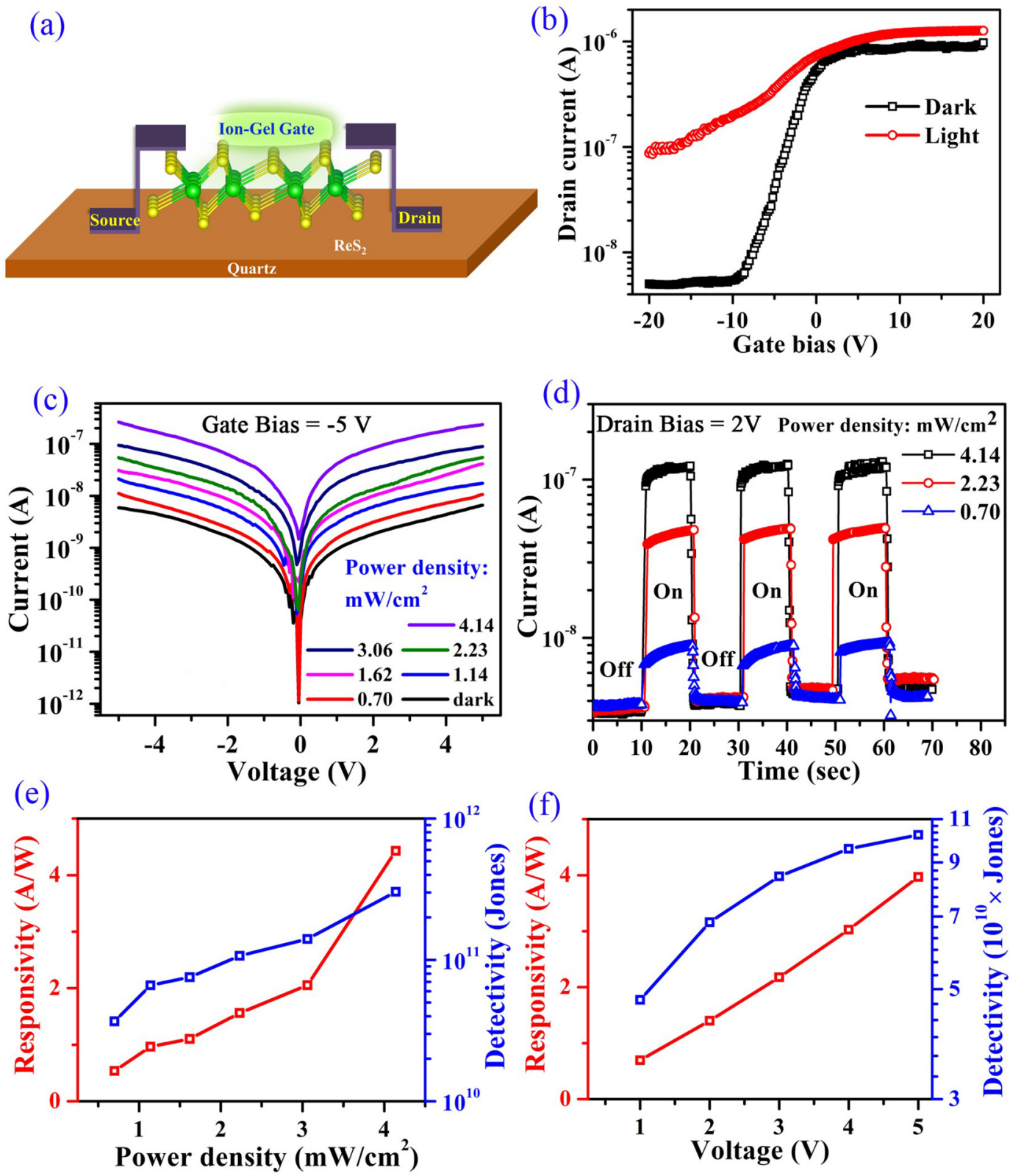

FIG. 10. Phototransistor characteristics of $\mathrm{ReS}_{2}$ sample F2: (a) schematic device diagram, (b) transfer characteristics in dark and illuminated conditions, (c) current vs voltage photo-response under dark and different illumination conditions, (d) photo-transistor switching characteristics with different illuminating power, (e) responsivity and detectivity with respect to the illuminated power density of light at a gate bias of $5 \mathrm{~V}$, and (f) responsivity and detectivity of the system as a function of voltage.

this shift can be explained by the concomitant increase of the electron-electron repulsion with the delay time, causing an increase of the required energy to generate an electron-hole pair. Interestingly, for F2, this shift is negligible, and thus, the optimized sample $\mathrm{F} 2$ produces almost constant energy exciton peaks with significantly long lifetimes.

In the next section, we establish the application of the vertically grown systems in the field of optoelectronic devices.

\section{OPTO-ELECTRONIC DEVICE APPLICATION}

We demonstrate the potential of the vertically grown optimized system F2 in the field of opto-electronics, where phototransistors were fabricated from F2 after using an ion- gel dielectric gate. The schematic and the corresponding characteristics of the device are depicted in Figs. 10(a)-10(f). For modern age integrated electronics, the ion-gel dielectrics are advantageous over the conventional solid state dielectrics in terms of their simple fabrication protocol at room temperature, cost effectiveness, low operational voltage, low power consumption, and the flexible choice of substrates. Figure 10(a) presents the schematic diagram of the field-effect transistor (FET), whereas in our practical system, the singlelayer electronic material as shown in the figure is replaced by the multilayered and vertically grown system F2. Figure 10(b) depicts the transfer characteristics presenting the drain-current $\left(I_{d}\right)$ vs gate voltage $\left(V_{g}\right)$ curves measured at the drain-source bias $\left(V_{d}\right)$ of $1 V$, exhibiting the typical FET behavior with 
distinct on-off states with a signature of $n$-type channel characteristics. The typical on-off ratio measured for such devices is $\sim 10^{2}$ with the threshold voltage $\left(V_{\mathrm{Th}}\right)$ being $\sim-8 \mathrm{~V}$. The low threshold voltage and the small operating voltage window ( -8 to $3 \mathrm{~V}$ ) for on-off switching are due to the high specific capacitance of the dielectric ion-gel, allowing an easy modulation of the work function of the $\mathrm{ReS}_{2}$ channel. Details of this measurement procedure and the extracted parameters are described in the Supplemental Material [37]. The field-effect mobility, as estimated for the $\operatorname{ReS}_{2}$ channel is $\sim 0.11 \mathrm{~cm}^{2} \mathrm{~V}^{-1} \mathrm{~s}^{-1}$, which is comparable with the earlier reported literature [58]. For the as-grown vertically oriented films, the carrier transport is a complicated process. In the present case, the active channel length of the photodetector is $\sim 2 \mathrm{~mm}$, which being much larger than the nanolithographically fabricated devices, results in a smaller value of the longitudinal electric field. Therefore, for such large-area devices, higher source-drain bias is commonly required for an efficient transport of photo-generated carriers.

The current-voltage $\left(I_{d}-V_{d}\right)$ characteristics as measured for fixed gate voltage $\left(V_{g}=-5 \mathrm{~V}\right)$ under different illumination conditions, are presented in Fig. 10(c), which indicate the symmetric $I-V$ nature as originated from the back-toback Schottky junction of the $\mathrm{Au}$ and $\mathrm{ReS}_{2}$ interface at the source and drain contacts. The significant rise of the current level upon illumination is attributed to the generation of photo-carriers, leading to a light-induced enhancement of the field-effect mobility of the channel. The temporal current response of the fabricated photodetector, upon periodic illuminations, has been recorded at $V_{d}=2 \mathrm{~V}$ and $V_{g}=-5 \mathrm{~V}$ for various illumination intensities, and the results are exhibited in Fig. 10(d). The rapid and periodic change in the current level for dark and illuminated conditions represents an excellent reproducibility and stability of the photodetectors. The results also demonstrate the absence of persistence photocurrent and fluctuation in the current level with time. The monotonic increase of responsivity $(R)$ and detectivity $(D)$ as a function of the intensity of illumination, as computed from the relation $R(\lambda)=\frac{J_{\text {Photo }}(\lambda)}{P_{d}}$ and $D=\frac{R(\lambda)}{\sqrt{q J_{\text {dark }}}}$, with $J_{\text {Photo }}$ and $J_{\text {dark }}$ being the photocurrent density in illuminated and dark conditions, $P_{d}$ is the incident power density, and $q$ is the electronic charge, is plotted in Fig. 10(e). The maximum photo-responsivity was recorded as $5 \mathrm{~A} / \mathrm{W}$, while the detectivity was recorded as high as $\sim 5 \times 10^{11}$ Jones, for $4.14 \mathrm{~mW} / \mathrm{cm}^{2}$ irradiation. The variation of both $R$ and $D$ with higher incident optical intensity becomes almost linear, suggesting that, with increasing power density, the rate of photo-induced carrier generation is proportional to the absorbed photon flux. While the variation of $R$ is linear, $D$ undergoes saturation with respect to the bias voltage.
Therefore, our results provide an important step toward the realization of the application of the vertically grown CVD $\mathrm{ReS}_{2}$ films as an efficient millimeter scale top-gate FET-based photodetector, for low-power applications in comparison with other vertically grown 2D systems $[59,60]$.

\section{CONCLUSIONS}

With a detailed theoretical comparison of the available structures in the literature, we have identified the proper structure of $\mathrm{ReS}_{2}$, capable of resolving the prior disputes about the correlation of its structure and electronic properties. Subsequently, with an appropriate bottom-up procedure, we have optimized the CVD growth conditions to obtain the sample with uniform vertical growth morphology. Microstructural and vibrational characterization identified this structure to be like our theoretically concluded one. The static and excited state optical properties of the films divulge a significantly long lifetime for the weakly bound excitons due to the presence of SV-induced mid-gap localization. We have demonstrated an application of these large-area vertically grown films by fabricating a photodetector with large responsivity and detectivity. The results presented in this paper will be useful for the future application of vertically grown systems in the fields of optics and opto-electronics.

\section{ACKNOWLEDGMENTS}

T.K.M. acknowledges the support of Department of Science and Technology, India for the INSPIRE Research Fellowship and S N Bose National Centre for Basic Sciences, Kolkata, India for funding. D.K. would like to acknowledge the BARC ANUPAM supercomputing facility for computational resources, BRNS CRP on Graphene Analogues for support and motivation, SAIF-IITB for TEM instrument, Madangopal Krishnan, and Abhijit Ghosh for help in accessing the departmental facilities.

J.R.G., A.K., and R.H. participated in CVD deposition. T.K.M. and A.K. plotted the figures and participated in analyzing both theoretical and experimental data. A.K. and K.V.A. did the TPA measurements and the corresponding data analysis. S.M. and S.K.R. performed the device fabrication and measurements. R.A. and K.D. helped in Raman measurement. A.M.R.S. and A.N. did the XPS and profilometry. S.D. and K.M. measured the PL. D.K. wrote the manuscript, performed DFT and TDDFT calculations, and participated in measurements and data analysis.
[1] S. Tongay, H. Sahin, C. Ko, A. Luce, W. Fan, K. Liu, J. Zhou, Y.-S. Huang, C.-H. Ho, J. Yan, D. F. Ogletree, S. Aloni, J. Ji, S. Li, J. Li, F. M. Peeters, and J. Wu, Nat. Commun. 5, 3252 (2014).

[2] M. Hafeez, L. Gan, H. Li, Y. Ma, and T. Zhai, Adv. Funct. Mater. 26, 4551 (2016).
[3] J. Lim, D. Jeon, S. Lee, J. S. Yu, and S. Lee, Nanotechnology 31, 115603 (2019).

[4] E. Zhang, Y. Jin, X. Yuan, W. Wang, C. Zhang, L. Tang, S. Liu, P. Zhou, W. Hu, and F. Xiu, Adv. Funct. Mater. 25, 4076 (2015).

[5] E. Liu, M. Long, J. Zeng, W. Luo, Y. Wang, Y. Pan, W. Zhou, B. Wang, W. Hu, Z. Ni, Y. You, X. Zhang, S. Qin, Y. Shi, K. 
Watanabe, T. Taniguchi, H. Yuan, H. Y. Hwang, Y. Cui, F. Miao, and D. Xing, Adv. Funct. Mater. 26, 1938 (2016).

[6] A. Dathbun, Y. Kim, S. Kim, Y. Yoo, M. S. Kang, C. Lee, and J. H. Cho, Nano Lett. 17, 2999 (2017).

[7] C. M. Corbet, C. McClellan, A. Rai, S. S. Sonde, E. Tutuc, and S. K. Banerjee, ACS Nano 9, 363 (2015).

[8] J. Kwon, Y. Shin, H. Kwon, J. Y. Lee, H. Park, K. Watanabe, T. Taniguchi, J. Kim, C.-H. Lee, S. Im, and G.-H. Lee, Sci. Rep. 9, 10354 (2019).

[9] H. Liu, B. Xu, J. M. Liu, J. Yin, F. Miao, C.-G. Duan, and X. G. Wan, Phys. Chem. Chem. Phys. 18, 14222 (2016).

[10] Q. Zhang, W. Wang, J. Zhang, X. Zhu, Q. Zhang, Y. Zhang, Z. Ren, S. Song, J. Wang, Z. Ying, R. Wang, X. Qiu, T. Peng, and L. Fu, Adv. Mater. 30, 1707123 (2018).

[11] O. B. Aslan, D. A. Chenet, A. M. van der Zande, J. C. Hone, and T. F. Heinz, ACS Photonics 3, 96 (2016).

[12] K. Wu, B. Chen, S. Yang, G. Wang, W. Kong, H. Cai, T. Aoki, E. Soignard, X. Marie, A. Yano, A. Suslu, B. Urbaszek, and S. Tongay, Nano Lett. 16, 5888 (2016).

[13] Y. Xiong, H. Chen, D. W. Zhang, and P. Zhou, Phys. Status Solidi RRL 13, 1800658 (2019).

[14] S. Zhang, N. Mao, N. Zhang, J. Wu, L. Tong, and J. Zhang, ACS Nano 11, 10366 (2017).

[15] N. Zhang, J. Lin, S. Zhang, S. Zhang, X. Li, D. Liu, H. Xu, J. Zhang, and L. Tong, Nano Res. 12, 563 (2019).

[16] H.-X. Zhong, S. Gao, J.-J. Shi, and L. Yang, Phys. Rev. B 92, 115438 (2015).

[17] S. Sim, D. Lee, A. V. Trifonov, T. Kim, S. Cha, J. H. Sung, S. Cho, W. Shim, M.-H. Jo, and H. Choi, Nat. Commun. 9, 351 (2018).

[18] X. Wang, K. Shinokita, H. E. Lim, N. B. Mohamed, Y. Miyauchi, N. T. Cuong, S. Okada, and K. Matsuda, Adv. Func. Mater. 29, 1806169 (2019).

[19] X. Meng, Y. Zhou, K. Chen, R. H. Roberts, W. Wu, J.-F. Lin, R. T. Chen, X. Xu, and Y. Wang, Adv. Opt. Mater. 6, 1800137 (2018).

[20] A. Dhara, D. Chakrabarty, P. Das, A. K. Pattanayak, S. Paul, S. Mukherjee, and S. Dhara, Phys. Rev. B 102, 161404(R) (2020).

[21] F. Cui, X. Li, Q. Feng, J. Yin, L. Zhou, D. Liu, K. Liu, X. He, X. Liang, S. Liu, Z. Lei, Z. Liu, H. Peng, J. Zhang, J. Kong, and H. Xu, Nano Res. 10, 2732 (2017).

[22] H. Jang, C. R. Ryder, J. D. Wood, M. C. Hersam, and D. G. Cahill, Adv. Mater. 29, 1700650 (2017).

[23] R. Oliva, M. Laurien, F. Dybala, J. Kopaczek, Y. Qin, S. Tongay, O. Rubel, and R. Kudrawiec, npj 2D Mater Appl. 3, 20 (2019).

[24] H. H. Murray, S. P. Kelty, R. R. Chianelli, and C. S. Day, Inorg. Chem. 33, 4418 (1994).

[25] J. C. Wildervanck and F. Jellinek, J. Less Common Met. 24, 73 (1971).

[26] C. H. Ho, Y. S. Huang, P. C. Liao, and K. K. Tiong, J. Phys. Chem. Solids 60, 1797 (1999).

[27] Z. Guo, A. Wei, Y. Zhao, L. Tao, Y. Yang, Z. Zheng, D. Luo, J. Liu, and J. Li, Appl. Phys. Lett. 114, 153102 (2019).

[28] P. Nagler, G. Plechinger, C. Schüller, and T. Korn, Phys. Status Solidi RRL 10, 185 (2016).

[29] N. B. Mohamed, K. Shinokita, X. Wang, H. E. Lim, D. Tan, Y. Miyauchi, and K. Matsuda, Appl. Phys. Lett. 113, 121112 (2018).

[30] M. Gehlmann, I. Aguilera, G. Bihlmayer, S. Nemšák, P. Nagler, P. Gospodarič, G. Zamborlini, M. Eschbach, V. Feyer, F.
Kronast, E. Młyńczak, T. Korn, L. Plucinski, C. Schüller, S. Blügel, and C. M. Schneider, Nano Lett. 17, 5187 (2017).

[31] D. Biswas, A. M. Ganose, R. Yano, J. M. Riley, L. Bawden, O. J. Clark, J. Feng, L. Collins-Mcintyre, M. T. Sajjad, W. Meevasana, T. K. Kim, M. Hoesch, J. E. Rault, T. Sasagawa, D. O. Scanlon, and P. D. C. King, Phys. Rev. B 96, 085205 (2017).

[32] J. L. Webb, L. S. Hart, D. Wolverson, C. Chen, J. Avila, and M. C. Asensio, Phys. Rev. B 96, 115205 (2017).

[33] R. He, J.-A. Yan, Z. Yin, Z. Ye, G. Ye, J. Cheng, J. Li, and C. H Lui, Nano Lett. 16, 1404 (2016).

[34] S. Yang, Y. Liu, M. Wu, L.-D. Zhao, Z. Lin, H.-c. Cheng, Y. Wang, C. Jiang, S.-H. Wei, L. Huang, Y. Huang, and X. Duan, Nano Res. 11, 554 (2018).

[35] X.-F. Qiao, J.-B. Wu, L. Zhou, J. Qiao, W. Shi, T. Chen, X. Zhang, J. Zhang, W. Ji, and P.-H. Tan, Nanoscale 8, 8324 (2016).

[36] H. J. Lamfers, A. Meetsma, G. A. Wiegers, and J. L. de Boer, J. Alloy Compd. 241, 34 (1996).

[37] See Supplemental Material at http://link.aps.org/supplemental/ 10.1103/PhysRevMaterials.5.054006 for details of the acquired methodologies for DFT and TDDFT calculations, anisotropic optical absorbance, details of CVD process and description of device fabrication and electrical measurements, description of PL and TA measurements. It also includes additional Refs. [61-64].

[38] J. H. Jung, C.-H. Park, and J. Ihm, Nano Lett. 18, 2759 (2018).

[39] Q. Jing, H. Zhang, H. Huang, X. Fan, Y. Zhang, X. Hou, Q. Xu, Z. Ni, and T. Qiu, Nanotechnology 30, 184001 (2019).

[40] X. Xu, Y. Guo, Q. Zhao, K. Si, Y. Zhou, J. Ma, J. Bai, and X. $\mathrm{Xu}$, Mater. Des. 159, 11 (2018).

[41] S. Horzum, D. Çakır, J. Suh, S. Tongay, Y.-S. Huang, C.-H. Ho, J. Wu, H. Sahin, and F. M. Peeters, Phys. Rev. B 89, 155433 (2014).

[42] X. Su, B. Zhang, Y. Wang, G. He, G. Li, N. Lin, K. Yang, J. He, and S. Liu, Photonics Res. 6, 498 (2018).

[43] D. Karmakar, R. Halder, N. Padma, G. Abraham, K. Vaibhav, M. Ghosh, M. Kaur, D. Bhattacharya, and T. V. C. Rao, J. Appl. Phys. 117, 135701 (2015).

[44] B. Jariwala, D. Voiry, A. Jindal, B. A. Chalke, R. Bapat, A. Thamizhavel, M. Chhowalla, M. Deshmukh, and A. Bhattacharya, Chem. Mater. 28, 3352 (2016).

[45] S. Jiang, M. Hong, W. Wei, L. Zhao, N. Zhang, Z. Zhang, P. Yang, N. Gao, X. Zhou, and C. Xie, Commun. Chem. 1, 17 (2018).

[46] K. Keyshar, Y. Gong, G. Ye, G. Brunetto, W. Zhou, D. P. Cole, K. Hackenberg, Y. He, L. Machado, and M. Kabbani, Adv. Mater. 27, 4640 (2015).

[47] Y. Kim, B. Kang, Y. Choi, J. H. Cho, and C. Lee, 2D Mater. 4, 025057 (2017).

[48] D. Ghoshal, A. Yoshimura, T. Gupta, A. House, S. Basu, Y. Chen, T. Wang, Y. Yang, W. Shou, J. A. Hachtel, J. C. Idrobo, T.-M. Lu, S. Basuray, V. Meunier, S.-F. Shi, and N. Koratkar, Adv. Func. Mater. 28, 1801286 (2018).

[49] X. Fang, Y. Gu, G. Yang, H. Bin, X. Ni, Q. Fan, X. Gu, Z. Dai, Y. Ding, and Y. Zhang, Mater. Res. Express 6, 0850e4 (2019).

[50] J. Borowiec, W. P. Gillin, M. A. C. Willis, F. S. Boi, Y. He, J. Q. Wen, S. L. Wang, and L. Schulz, J. Phys. Condens. Matter 30, 055702 (2018). 
[51] F. Qi, Y. Chen, B. Zheng, J. He, Q. Li, X. Wang, B. Yu, J. Lin, J. Zhou, P. Li, and W. Zhang, J. Mater. Sci. 52, 3622 (2017).

[52] X. He, F. Liu, P. Hu, W. Fu, X. Wang, Q. Zeng, W. Zhao, and Z. Liu, Small 11, 5423 (2015).

[53] V. Srinivas, S. K. Barik, B. Bodo, D. Karmakar, and T. V. Chandrasekhar Rao, J. Magn. Magn. Mater. 320, 788 (2008).

[54] K. Zhao, F. Huang, C.-M. Dai, W. Li, S.-Y. Chen, K. Jiang, Y.-P. Huang, Z. Hu, and J. Chu, J. Phys. Chem. C 122, 29464 (2018).

[55] K. Xu, H.-X. Deng, Z. Wang, Y. Huang, F. Wang, S.-S. Li, J.-W. Luo, and J. He, Nanoscale 7, 15757 (2015).

[56] T. K. Maji, A. J. R, S. Mukherjee, R. Alexander, A. Mondal, S. Das, R. K. Sharma, N. K. Chakraborty, K. Dasgupta, A. M. R. Sharma, R. Hawaldar, M. Pandey, A. Naik, K. Majumdar, S. K. Pal, K. V. Adarsh, S. K. Ray, and D. Karmakar, ACS Appl. Mater. Interfaces 12, 44345 (2020).
[57] T. K. Maji, K. Vaibhav, S. K. Pal, K. Majumdar, K. V. Adarsh, and D. Karmakar, Phys. Rev. B 99, 115309 (2019).

[58] B. Chamlagain, Q. Li, N. J. Ghimire, H.-J. Chuang, M. M. Perera, H. Tu, Y. Xu, M. Pan, D. Xaio, J. Yan, D. Mandrus, and Z. Zhou, ACS Nano 8, 5079 (2014).

[59] J. Y. Park, H.-E. Joe, H. S. Yoon, S. Yoo, T. Kim, K. Kang, B.-K. Min, and S. C. Jun, ACS Appl. Mater. Interfaces 9, 26325 (2017).

[60] B. Rahmati, I. Hajzadeh, R. Karimzadeh, and S. M. Mohseni, Appl. Surf. Sci. 455, 876 (2018).

[61] S. Smidstrup, D. Stradi, J. Wellendorff, P. A. Khomyakov, U. G. Vej-Hansen, M.-E. Lee, T. Ghosh, E. Jónsson, H. Jónsson, and K. Stokbro, Phys. Rev. B 96, 195309 (2017).

[62] S. Smidstrup et al., J. Phys. Cond. Matter 32, 015901 (2019).

[63] S. Grimme, WIREs Comput. Mol. Sci. 1, 211 (2011).

[64] http://elk.sourceforge.net/. 